\title{
Moduli space actions on the Hochschild co-chains of a Frobenius algebra I: cell operads
}

\author{
Ralph M. Kaufmann
}

\begin{abstract}
This is the first of two papers in which we prove that a cell model of the moduli space of curves with marked points and tangent vectors at the marked points acts on the Hochschild co-chains of a Frobenius algebra. We also prove that a there is dg-PROP action of a version of Sullivan chord diagrams which acts on the normalized Hochschild co-chains of a Frobenius algebra. These actions lift to operadic correlation functions on the co-cycles. In particular, the PROP action gives an action on the homology of a loop space of a compact simply-connected manifold.

In this first part, we set up the topological operads/PROPs and their cell models. The main theorems of this part are: There is a cell model operad for the moduli space of genus $g$ curves with $n$ punctures and a tangent vector at each of these punctures, there exists a $\mathrm{CW}$ complex whose chains are isomorphic to a certain type of Sullivan chord diagrams and they form a PROP. Furthermore there exist weak versions of these structures on the topological level which all lie inside an all encompassing cyclic (rational) operad.
\end{abstract}

Mathematics Subject Classification (2000). 55P48; 16E40, 17Axx, 18D50, 32G15, 32G81, $81 \mathrm{~T} 40$.

Keywords. Moduli spaces, operads, Hochschild cohomology, foliations, cell models, string topology, Sullivan chord diagrams, PROPs, conformal field theory.

\section{Introduction}

Recently the operations of cells on the Hochschild complex of associative algebras have been intensely studied. There are three main sources for this interest. The first is Deligne's conjecture which has been solved in various ways [Ko3], [T], [MS1], [MS2], [MS3], [V], [KS1], [MS2], [BF], [K2] together with its generalization to $A_{\infty}$-algebras and to the cyclic case using the framed little discs operad [K3] in conjunction with Frobenius algebras. The second source is the string topology of Chas and Sullivan [CS], [V2], [CJ], [C], [CG], [Ch], [Me], [S1], [S2], and lastly the third source are considerations of $D$-branes in open/closed string theory as deformations of the Hochschild complex see e.g. [KR], [KLi1], [KLi2] ${ }^{1}$. See also [KS2], [Co]

\footnotetext{
${ }^{1} \mathrm{~A}$ more extensive discussion of these links is given in [K4].
} 
for discussions of these topics in different settings. The exact relationship of these constructions to ours is at present not known.

In this paper and its sequel [K4] we prove the following:

Theorem A. The moduli space $M_{g, n+1}^{1^{n+1}}$ of genus $g$ curves with $n$ punctures and a tangent vector at each of these punctures has the structure of a rational cyclic operad. This structure induces a cyclic dg operad structure on a cell model computing the cohomology of $M_{g, n+1}^{1^{n+1}}$.

Furthermore the cell level operad operates on the Hochschild co-chains of a Frobenius algebra. It also yields correlation functions on the tensor algebra of the co-cycles of a differential algebra $(A, d)$ with a cyclically invariant trace $\int: A \rightarrow k$ which satisfies $\int d a=0$ and whose induced pairing on $H=H(A, d)$ turns $H$ into a Frobenius algebra.

The first part of the theorem together with the graph description of this moduli space given below can be taken to say that we define a combinatorial version of conformal field theory.

Furthermore, there is also a PROPic version of this action related to string topology.

Theorem B. There is a rational topological quasi-PROP which is homotopic to a $C W$ complex whose cellular chains are isomorphic as a free Abelian group to a certain type of Sullivan chord diagrams. These chains form a dg-PROP and hence induce this structure on the chord diagrams. Furthermore if $H$ is a Frobenius algebra there is a PROPic action on the Hochschild co-chains of $H$ that is a dg action. This $d g$ action of a dg-PROP on the dg algebra of Hochschild co-chains naturally descends to an action of the homology of the CW-complex on the Hochschild cohomology of a Frobenius algebra.

Moreover for $\left(A, d, \int, H\right)$ as in Theorem $A$ the action on $H$ is induced by correlation functions on the tensor algebra of $A$ that yield operadic correlation functions on the tensor algebra of the co-cycles of $A$ for any $(A, d)$ as above.

Finally, the $B V$ operator, which is given by the action of the sub-PROP equivalent to the framed little discs operad, acts as in [K3]. Thus the BV operator for the action on the Hochschild cohomology of $H$ is identified with Connes'operator $B$ under the identification of the Hochschild cohomology of a Frobenius algebra with its cyclic cohomology of $H$.

The notions "quasi" and "rational" denote certain weakenings of the axioms which are explained below. The notion of quasi-operad and its uses have been discussed in [K1]. A thorough analysis of rational operads which we introduce here for the first time and the structures they can induce on homology and the chain level is unfortunately beyond the scope of this article and will have to be relegated to future work. The definition of the new notion of operadic correlation functions is contained 
in the second part [K4]. This notion should be thought of as the correct definition of a $\mathrm{dg}$ algebra $(A, d)$ over a cyclic operad. It is the mathematical incarnation of the fact that OPEs in physics are actually only defined within correlators and only on BRST closed fields. An immediate consequence using Jones' [J] cyclic description of the free loop spaces and its $S^{1}$-action then is the following

Corollary. When taking field coefficients, the above action gives a dg action of a dgPROP of Sullivan chord diagrams on the $E^{1}$-term of a spectral sequence converging to $H_{*}(L M)$, that is to the homology of the loop space of a simply connected compact manifold, and hence induces operations on the homology of the loop space.

Lastly, there is a version of these statements in the case where $(V, d)$ is a vector space with a differential and a pairing $\langle$,$\rangle that is symmetric such that \langle d v, w\rangle+$ $\langle v, d w\rangle=0$ for all $v, w \in V$ and $\langle$,$\rangle is non-degenerate on H=H(V, d)$, which is finite dimensional.

Theorem C. The operads and PROPs above also act on the tensor algebra $T V$ of a triple $(V, d,\langle\rangle$,$) as specified in the preceding paragraph and yield operadic$ correlation functions for the co-cycles of $T V$.

This action is different from the algebra case of Theorems A and B, making the result interesting in its own right. In the algebra case, the action involves the algebra multiplication, while the present action works in the setting of only a vector space. Furthermore, this action "descends" to an action of the stabilized arc operad, which forms a spectrum [K5].

The proof of these facts consists mainly of two steps. First defining the respective topological objects and then defining their actions.

The first step which is the content of this paper is actually quite involved, since there are several natural generalizations of the operads Cact and Cacti (see [K1]) yielding the Gerstenhaber and BV structures and several types of chain level models for them. The most notable ones are the Arc operad of [KLP] and its subspace $\mathcal{A} r c_{\#}^{0}$ consisting of quasi-filling arc families on surfaces without punctures. We prove below that the space $\mathcal{A} r c_{\#}^{0}$ is isomorphic to the moduli space of genus $g$ curves with $n$ punctures and a tangent vector at each of these punctures modded out by the action of $\mathbb{R}_{>0}$ which scales all the tangent vectors simultaneously. Other generalizations are isomorphic to different versions of metric Sullivan chord diagrams as we explain below.

The reader who is mainly interested in how the action is defined and wants to forgo the geometric topological and algebraic topological constructions of the various relevant operads and PROP can skip ahead to the second part [K4].

In this part, we will systematically extend and augment the operad structure of Arc and use these topological structures to induce operations on the chain level. The 
results are novel operadic and PROPic structures associated to various restrictions and extensions of the moduli space $M_{g, n+1}^{1^{n+1}}$ on the topological and chain level. The elements of $\mathcal{A} r c$ or better of its ambient CW complex $\mathcal{A}$ can be thought of as metric graphs on a surface with boundaries and one marked point on each boundary, where the set of these points is equal to the set of the vertices of the graph. A $r c$ is then the subset where all boundaries are "hit" that is none of the vertices has valence 0 . Although $\mathcal{A} r c$ is an operad, $\mathcal{A} r c_{\#}^{0}$ is only an operad on a dense subset. The cause of this is that on a codimension 1 subset the gluing defined in the ambient $\mathcal{A} r c$ will lead outside of $\mathcal{A} r c_{\#}^{0}$. Thus we are lead to consider rational topological operads, that is operads whose gluings are defined on dense open subsets. Since the "bad" part is of codimension at least one, this structure, however, descends to a true operad structure on the chain level for suitable chains. Considering the dg structure of the PROP action, we will see in [K4] that we also have to extend the gluings of the $\mathcal{A} r c$ operad to its ambient $\mathrm{CW}$ complex $\mathcal{A}$ (we give these constructions in $\S 2$ ).

In order to go into the PROP framework, we need to distinguish the boundaries as "in" and "out" boundaries. The resulting space which accommodates the extra markings is denoted by $A r^{i / o}$. There are natural additional restrictions which can be imposed on the graphs. The first is allowing edges only between "in" and "out"; this space will be called $\mathcal{A} r c^{i \leftrightarrow o}$. Only barring edges from "in" to "in", we obtain a subspace which will be called $\mathcal{A} r c^{i \leftrightarrow i}$. These spaces naturally form di-operads. In the dual graph terminology they correspond to various generalizations of Sullivan chord diagrams. We include a discussion of several versions of Sullivan chord diagrams along with their dual representation within $\mathcal{A} r c$ that appear in this context and in the literature [CS], [S1], [S2], [CG], [TZ], see $§ 1.4$. When trying to upgrade them to PROPs one has to allow the gluing of all "in" boundaries to all "out" boundaries. This poses too many conditions to make the gluing associative on the topological level. As in [K1] this situation can be remedied by weakening the notion of a PROP to that of a quasi-PROP that is a PROP in which the associativity holds up to homotopy. The largest sub-di-operad of $\mathcal{A} r c^{i / o}$ for which this strategy works is $\mathcal{A} r c^{i \leftrightarrow i}$. For suitable chains the induced chain level structure is a PROP in the strict sense. The subspace of $\mathcal{A} r c^{i / o}$ given by those elements whose arcs only run from "in" to "out" but also all "in" boundaries are hit is called $\overline{\mathcal{A r c}}^{i} \leftrightarrow$.

There is an even finer structure than the "in/out" distinction which is given by an angle marking. Using angle markings we obtain an all encompassing rational operad. Here the angles refer to the angles of the arc graphs which define elements of the A $r c$ operad and the marking takes values in $\mathbb{Z} / 2 \mathbb{Z}$. The space of the elements of $\mathcal{A} r c$ together with an angle marking is called $\mathcal{A} r c^{\perp}$. Keeping with the theme of angle markings we define the space $\mathcal{A}^{\angle}$ to be the space of all elements of $\mathcal{A}$ with additional angle markings.

Armed with these notions we can state a first omnibus theorem summing up essential results for the topological objects of interest. 
Theorem D. We have the following topological structures:

(1) The subspace A $r_{\#}^{0}$ of Arc is a cyclic rational topological operad. It induces the structure of a cyclic rational topological operad on the spaces $M_{g, n+1}^{1^{n+1}}$.

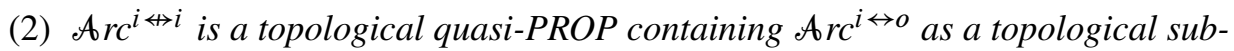
quasi-PROP. It induces quasi-PROP structures on the respective versions of metric Sullivan chord diagrams.

(3) A $r c^{<}$is a cyclic rational topological operad. It "contains" all of the above in the sense that all the structures can be derived from the gluing in $\mathrm{Arc}^{<}$.

(4) $\overline{A A r c}^{i \leftrightarrow o}$ is a quasi-PROP structure extending that of $\mathcal{A} r c^{i \leftrightarrow o}$. It induces a quasiPROP structure on the extension of Sullivan chord diagrams for which we define the action on the Hochschild co-chains in the second part [K4].

(5) There exists a CW complex $\overline{\mathcal{A r c}}_{1}^{i \leftrightarrow o}$ which is homotopy equivalent to $\overline{\mathcal{A r c}}^{i \leftrightarrow o}$. It can be endowed with the structure of a quasi-PROP that is homotopic to the quasi-PROP structure of $\overline{\mathcal{A r c}}^{i \leftrightarrow o}$.

The same results hold for the restrictions to surfaces with no punctures and to genus zero surfaces as well as the intersection of these conditions.

The main spaces of interest in the sequel will be $\mathcal{A} r c_{\#}^{0}$ and $\overline{\mathcal{A} r c}^{i \leftrightarrow o}$. As stated above the latter has a CW-model $\overline{A r c}_{1}^{i \leftrightarrow o}$. This model is the generalization of acti $^{1}$ of [K1] to the PROP setting. The space $\mathcal{A} r c_{\#}^{0}$ yields the moduli-space operations expected for instance from a $D$-brane point of view and $\overline{\mathcal{A} r c}^{i \leftrightarrow o}$ yields String Topology type operations, i.e. an PROPic operation of an extension of Sullivan chord diagrams on the loop space of a simply connected manifold. Here we use the term Sullivan chord diagram for the contracted version Sullivan chord diagrams; that is that type of diagrams which one obtains after contracting ghost edges.

We would also like to mention that there is a generalization $\overline{\mathcal{A r c c}}$ i↔i of $\overline{\mathcal{A r c}} i \leftrightarrow o$ where one is also allowed to have arcs running from "out" to "out". This is again a quasi-PROP whose cells give a PROP. It is the generalization of $\mathcal{A} r c^{i \leftrightarrow i}$. It turns out, see [K4] that although there is a natural action on the Hochschild co-chains, the action

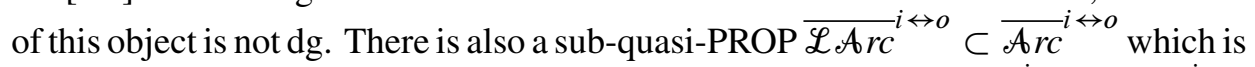
the generalization of the spineless cacti and a sub-CW complex $\overline{\mathscr{L} A r c}_{1}^{i \leftrightarrow o} \subset \overline{\mathcal{A r c}}_{1}^{i \leftrightarrow o}$ corresponding to it. In [K4] we show that there is a natural action of $\overline{\mathscr{L A C r c}}_{1}^{i \leftrightarrow o}$ that generalizes the $\Xi_{2}$ action of [MS3].

There is a global object giving rise to all of the needed structures. This is the $\mathrm{CW}$-complex $\mathfrak{A}^{<}$of angle marked metric arc graphs. This space has the structure of a rational-quasi-modular-operad. Although this is a weak structure when restricted to the different subspaces which we pick out, it induces the structures of cyclic operads, 
rational operads, quasi-PROPs, etc. we discussed above. In particular this weak structure is more rigid on the cell level and the correlators that induce all the different actions are defined for the cells of $\mathcal{A}^{<}$[K4].

The next step is to form cell models for the spaces in question for which topological structure induce the honest (not "quasi" or "rational") structures on the chain level. An essential tool we use is the filtration on $\mathcal{A}$ by the number of edges of the graph, which gives a filtration of the cells of $\mathcal{A}$. The gluing rules in $\mathcal{A} r c$ respect this filtration, so it is possible to consider the associated graded on the cell level. This for instance gets rid of the codimension one parts which are responsible for the fact that $\mathcal{A r c} \#$ is only a rational operad.

The essential results used in the sequel for the cell level can be summed up as follows.

Theorem E. We have the following chain level structures:

(1) The relative cellular chains of $\mathcal{A} r c$ in $\mathcal{A}$ form an operad $\mathcal{C}_{o}^{*}(\mathcal{A r c})$ which is filtered by the dimension of the chains. The associated graded $\mathrm{Gr} \mathcal{C}_{o}^{*}(\mathcal{A r c})$ is also a cyclic operad.

(2) The suboperad $\mathrm{Gr} \complement_{o}^{*}\left(\mathcal{A} r c_{\#}\right)$ is a dg operad. This operad is an operad structure on the collection of graph-complex-models of the moduli spaces $M_{g, n+1}^{1^{n+1}}$ which calculates their cohomology.

(3) $C C_{*}\left(\mathcal{A}^{L}\right)$ is an operad, which contains the relative chains of $\mathrm{Gr}_{\mathrm{o}}^{*}\left(\mathcal{A r c}^{<}\right)$. The chains $\mathrm{Gr} \mathcal{C}_{o}^{*}(\mathcal{A} r c)$ are also naturally embedded.

(4) $\mathcal{C}_{o}^{*}\left(\overline{\mathcal{A r c}}^{i \leftrightarrow o}\right)$ is a di-operad and a PROP. It is filtered by dimension and the associated graded $\operatorname{Gr} \mathcal{C}_{o}^{*}\left(\overline{\mathcal{A r c}}^{i \leftrightarrow o}\right)$ is also a di-operad and a PROP. This is a PROP of the version of Sullivan chord diagrams relevant for our purposes.

(5) The cellular chains $C C_{*}\left(\overline{A A r c}_{1}^{i \leftrightarrow o}\right)$ form a di-operad and a PROP - that is a chain model for $\overline{\mathcal{A r c}}^{i \leftrightarrow o}$. This is a cellular realization of the above PROP of Sullivan chord diagrams.

Notice that we have two types of complexes, those of relative chains, which can be thought of as pseudo-cells which we denote by $\ell_{o}^{*}$ and honest cellular chains of cell complexes which we denote by $C_{*}$. Again, the same results hold for the restrictions to surfaces with no punctures and to genus zero surfaces as well as the intersection of these conditions.

These cell models are related via the dual graph construction to the free Abelian groups generated by certain types of ribbon graphs, for instance: $\operatorname{Gr}_{o}^{*}\left(\mathcal{A} r c_{\#}^{0}\right) \cong$ $\mathcal{R} i b$, the space of marked ribbon graphs and $\mathcal{C}_{o}^{*}\left(\mathcal{A} r c_{\#}^{i \leftrightarrow o}\right) \cong \mathcal{R} i b^{i \leftrightarrow o}$ that is the perfectly partitioned di-graphs which can be thought of as stabilized Sullivan chord diagrams, see $\$ 1.4$. 
The paper is organized as follows.

In $\S 1$, we define the types of graphs and the operations on these graphs which we will need in the sequel. In $\$ 2$ we start by recalling the constructions of [KLP] albeit in slightly different language using mainly graphs. We go on to generalize these constructions and augment the setting by including a filtration on this space. One of the new results of this section is the construction of a filtered cell level operad built on $A r c$ and its associated graded. The third paragraph, §3, then details the identification of the subspace $\mathcal{A} r r_{\#}^{0}$ with the moduli space of surfaces with marked points and tangent vectors at the marked points. The tool here is the dual graph construction which turns an element of $\mathcal{A} r c_{\#}^{0}$ into a marked metric ribbon graph. The first main result of the paper, namely that the associated graded of the cells of $\mathcal{A} r c_{\#}^{0}$ forms an operad is included in $\S 4$. This paragraph also contains the identification of this complex with the relevant graph complex. The last paragraph $\$ 5$ contains the generalizations to the di-operad and PROP structures discussed in the Introduction. The main link between all the objects are angle marked arc graphs. The second main result which will be used in the sequel is the construction of cell and CW models for the graphs corresponding to Sullivan chord diagrams whose action will render the String Topology type operations.

Acknowledgments. We would like to thank the Max-Planck-Institute for Mathematics where this work was started, a good portion of it was written in the summer of 2005 and the finishing touches were put on in the summer of 2006. The two papers received their final form at the MSRI, which we would like to thank for its hospitality in May 2006. It is a pleasure to thank Bob Penner, Ralph Cohen, Jim McClure, Dev Sinha and Craig Westerland for discussions on various details during various stages of this project.

Conventions. We fix $k$ to be a field of arbitrary characteristic. We let $\bar{n}$ be the set $\{0, \ldots, n\}$.

\section{Graphs and ribbon graphs}

1.1. Classes of graphs. In this section, we formally introduce the graphs and the operations on graphs which we will use in our analysis.

1.1.1. Graphs. A graph $\Gamma$ is a tuple $\left(V_{\Gamma}, F_{\Gamma}, t_{\Gamma}: F_{\Gamma} \rightarrow F_{\Gamma}, \partial_{\Gamma}: F_{\Gamma} \rightarrow V_{\Gamma}\right)$ where $\imath_{\Gamma}$ is an involution $\imath_{\Gamma}^{2}=$ id without fixed points. We call $V_{\Gamma}$ the vertices of $\Gamma$ and $F_{\Gamma}$ the flags of $\Gamma$. The edges $E_{\Gamma}$ of $\Gamma$ are the orbits of the flags under the involution $l_{\Gamma}$. A directed edge is an edge together with an order of the two flags which define it. In case there is no risk of confusion, we will drop the subscripts $\Gamma$. Notice that $f \mapsto(f, l(f))$ gives a bijection between flags and directed edges. 
We also call $F_{\Gamma}(v):=\partial^{-1}(v) \subset F_{\Gamma}$ the set of flags of the vertex $v$ and call $\left|F_{v}(\Gamma)\right|$ the valence of $v$ and denote it by $\operatorname{val}(v)$. We also let $E(v)=\{\{f, l(f)\} \mid f \in F(v)\}$ and call these edges the edges incident to $v$.

The geometric realization of a graph is given by considering each flag as a half-edge and gluing the half-edges together using the involution $t$. This yields a one-dimensional $\mathrm{CW}$ complex whose realization we call the realization of the graph.

1.1.2. Ribbon graphs. A ribbon graph with tails is a connected graph together with a cyclic order of the set of flags of the vertex $F_{\Gamma}(v)$ for every vertex $v$. A ribbon graph with tails that satisfies $\operatorname{val}(v) \geq 2$ for all vertices $v$ will simply be called a ribbon graph. Notice that we do not fix val $(v) \geq 3$. We will call a ribbon graph stable if it does satisfy this condition.

For a ribbon graph with tails, the tail vertices are $V_{\text {tail }}=\left\{v \in V_{\Gamma} \mid \operatorname{val}(v)=1\right\}$, the tail edges $E_{\text {tail }}(\Gamma)$ are the edges incident to the tail vertices and the tail flags $F_{\text {tail }}(\Gamma)$ are those flags of the tail edges which are not incident to the tail vertices.

A graph with a cyclic order of the flags at each vertex gives rise to bijections $\curvearrowright_{v}: F_{v} \rightarrow F_{v}$ where $\curvearrowright_{v}(f)$ is the next flag in the cyclic order. Since $F=\bigsqcup F_{v}$ one obtains a map $\curvearrowright: F \rightarrow F$. The orbits of the map $N:=\curvearrowright \circ \imath$ are called the cycles or the boundaries of the graph. These sets have the induced cyclic order.

Notice that each boundary can be seen as a cyclic sequence of directed edges. The directions are as follows. Start with any flag $f$ in the orbit. In the geometric realization go along this half-edge starting from the vertex $\partial(f)$, continue along the second half-edge $l(f)$ until you reach the vertex $\partial(l(f))$ then continue starting along the flag $\curvearrowright(l(f))$ and repeat.

An angle is a pair of flags $(f, \curvearrowright(f))$, we denote the set of angles by $\angle_{\Gamma}$. It is clear that $f \mapsto(f, \curvearrowright(f))$ yields a bijection between $F_{\Gamma}$ and $\angle_{\Gamma}$. It is however convenient to keep both notions.

1.1.3. The genus of a ribbon graph and its surface. The genus $g(\Gamma)$ of a ribbon graph $\Gamma$ is given by $2-2 g(\Gamma)=\left|V_{\Gamma}\right|-\left|E_{\Gamma}\right|+\operatorname{Cyc}(\Gamma)=\chi(\Gamma)+\operatorname{Cyc}(\Gamma)$ where $\operatorname{Cyc}(\Gamma)=\#$ cycles.

The surface $\Sigma(\Gamma)$ of a ribbon graph $\Gamma$ is the surface obtained from the realization of $\Gamma$ by thickening the edges to ribbons, i.e., replace each 0 -simplex $v$ by a closed oriented disc $D(v)$ and each 1-simplex $e$ by $e \times I$ oriented in the standard fashion. Now glue the boundaries of $e \times I$ to the appropriate discs in their cyclic order according to the orientations. This is a surface whose boundary components are given by the cycles of $\Gamma$. The graph $\Gamma$ is naturally embedded as the spine of this surface $\Gamma \subset \Sigma(\Gamma)$. Let $\bar{\Sigma}(\Gamma)$ be the surface obtained from $\Sigma(\Gamma)$ by filling in the boundaries with discs. Notice that the genus of the $\bar{\Sigma}(\Gamma)$ is $g(\Gamma)$ and $\chi(\Gamma)=$ $2-2 g(\Sigma(\Gamma))$. 


\subsubsection{Marked ribbon graphs}

Definition 1.1. A ribbon graph together with a distinguished cycle $c_{0}$ is called treelike if

i) the graph is of genus 0 and

ii) for all flags either $f \in c_{0}$ or $l(f) \in c_{0}$ (and not both).

In other words each edge is traversed exactly once by the cycle $c_{0}$. Therefore there is a cyclic order on all (non-directed) edges, namely the cyclic order of $c_{0}$.

Definition 1.2. A marked ribbon graph is a ribbon graph together with a map mk: \{cycles $\} \rightarrow F_{\Gamma}$ satisfying the conditions:

i) For every cycle $c$ the directed edge $\mathrm{mk}(c)$ belongs to the cycle.

ii) All vertices of valence two are in the image of $\mathrm{mk}$, that is, $\operatorname{val}(v)=2$ for all $v$ implies $v \in \operatorname{Im}(\partial \circ \mathrm{mk})$.

Notice that on a marked treelike ribbon graph there is a linear order on each of the cycles $c_{i}$. This order is defined by upgrading the cyclic order to the linear order $\prec_{i}$ in which $\mathrm{mk}\left(c_{i}\right)$ is the smallest element.

1.1.5. Labelling and marking graphs. A labelling of the edges of a graph $\Gamma$ by a set $S$, is a map $E_{\Gamma} \rightarrow S$. A labelling of a ribbon graph $\Gamma$ by a set $S$ is a map Lab: $\{$ cycles of $\Gamma\} \rightarrow S$, we will write $c_{i}:=\mathrm{Lab}^{-1}(i)$. An angle marking by $S$ is a map $\mathrm{mk}^{\angle}: \angle_{\Gamma} \rightarrow S$.

Notation 1.3. We will write $\mathcal{R i}_{n, g}$ for the set of marked ribbon graphs of genus $g$ with $n$ boundary cycles and, by abuse of notation, also for the free Abelian group generated by this set.

We set $\mathcal{R} i b:=\bigsqcup_{n, g} \mathcal{R} i b_{n, g}$, and we will again not distinguish in notation between the set $\mathcal{R} i b$, the free Abelian group generated by it, and the set $\left\{\coprod_{g} \mathcal{R} i b_{n, g} \mid n \in \mathbb{N}\right\}$ to avoid unnecessary clutter. We also write $\mathcal{R} i b(n)$ for the set of marked ribbon graphs with $n+1$ cycles together with a labelling by $\{0, \ldots, n\}$ of these cycles. Again we also denote the free Abelian group generated by this set as $\mathcal{R} i b(n)$. Finally, to streamline the notation, we will denote the collection $\{\mathcal{R} i b(n) \mid n \in \mathbb{N}\}$ simply by $\mathcal{R} i b$.

The meaning of the symbols will always be clear from the context.

1.1.6. Spineless marked ribbon graphs. A marked treelike ribbon graph is called spineless if the following holds:

i) There is at most one vertex of valence 2. If there is such a vertex $v_{0}$ then $\partial\left(\operatorname{mk}\left(c_{0}\right)\right)=v_{0}$.

ii) The induced linear orders on the $c_{i}$ are (anti-)compatible with that of $c_{0}$, i.e. $f \prec_{i} f^{\prime}$ if and only if $l\left(f^{\prime}\right) \prec_{0} l(f)$. 
1.2. Operations on graphs. In this section, we will give the basic definitions of the operations on graphs which we will need.

1.2.1. Contracting edges. The contraction $\Gamma / e=\left(\bar{V}_{\Gamma}, \bar{F}_{\Gamma}, \bar{l}, \bar{\partial}\right)$ of a graph $\Gamma=$ $\left(V_{\Gamma}, F_{\Gamma}, l, \partial\right)$ with respect to an edge $e=\{f, l(f)\}$ is defined as follows. Let $\sim$ be the equivalence relation induced by $\partial(f) \sim \partial(l(f))$. Then let $\bar{V}_{\Gamma}:=V_{\Gamma} / \sim$, $\bar{F}_{\Gamma}=F_{\Gamma} \backslash\{f, \imath(f)\}$ and $\bar{\imath}: \bar{F}_{\Gamma} \rightarrow \bar{F}_{\Gamma}, \bar{\partial}: \bar{F}_{\Gamma} \rightarrow \bar{V}_{\Gamma}$ be the induced maps.

For a ribbon graph the cyclic order is the one which descends naturally.

For a marked ribbon graph, we define the marking of $\left(\bar{V}_{\Gamma}, \bar{F}_{\Gamma}, \bar{l}, \bar{\partial}\right)$ to be $\overline{\mathrm{mk}}(\bar{c})=$ $\overline{\mathrm{mk}(c)}$ if $\mathrm{mk}(c) \notin\{f, l(f)\}$ and $\overline{\mathrm{mk}}(\bar{c})=\overline{N \circ \iota(\operatorname{mk}(c))}$ if $\operatorname{mk}(c) \in\{f, l(f)\}$, viz. the image of the next flag in the cycle.

If there is an angle marking, set $f^{\prime}=N^{-1}(f), f^{\prime \prime}=\curvearrowright(f), g^{\prime}=N^{-1}(l(f))$ and $g^{\prime \prime}=\curvearrowright(l(f))$, let $\mathrm{mk}^{\perp}\left(f^{\prime}, f\right)=a, \mathrm{mk}^{\perp}\left(f, f^{\prime \prime}\right)=b, \mathrm{mk}^{\perp}\left(g^{\prime}, l(f)\right)=$ $c$ and $\mathrm{mk}^{\perp}\left(l(f), g^{\prime \prime}\right)=d$; after the contraction we set $\mathrm{mk}^{\perp}\left(f^{\prime}, g^{\prime \prime}\right)=\overline{\bar{a} \bar{d}}$ and $\mathrm{mk}^{\perp}\left(g^{\prime}, f^{\prime \prime}\right)=\overline{\bar{b}} \bar{c}$, where we use the notation $\bar{a}=1-a \in \mathbb{Z} / 2 \mathbb{Z}$.

1.2.2. Deleting edges. The graph $\Gamma \backslash e=\left(\bar{V}_{\Gamma}, \bar{F}_{\Gamma}, \bar{\imath}, \bar{\partial}\right)$ obtained by deletion of an edge $e$ of a graph $\Gamma=\left(V_{\Gamma}, F_{\Gamma}, \imath, \partial\right)$ with respect to an edge $e=\{f, l(f)\}$ is defined as follows: Set $\bar{V}_{\Gamma}=V_{\Gamma}, \bar{F}_{\Gamma}=F_{\Gamma} \backslash\{f, \imath(f)\}, \bar{\imath}=\left.\imath\right|_{\bar{F}_{\Gamma}}, \bar{\partial}=\left.\partial\right|_{\bar{F}_{\Gamma}}$, that is, delete the edge. Notice there might be left-over lone vertices if $e$ was the only edge of the respective vertices, also the graph might become disconnected.

If the graph was a ribbon graph the resulting graph again is a ribbon graph using the cyclic order that descends naturally. In the case of a marked ribbon graph, if there was a marking and say $f=\operatorname{mk}\left(c_{i}\right)$ for $f \in e$ then we set $\operatorname{mk}\left(c_{i}\right)=N^{-1}(f)$ to be the previous flag, otherwise, the marking also descends naturally.

If there is an angle marking and $f \in e$ with val $(\partial(f))>1$, with markings $\mathrm{mk}^{\angle}\left(f^{\prime}, f\right)=a, \mathrm{mk}^{\angle}\left(f, f^{\prime \prime}\right)=b$ for $f^{\prime}=N^{-1}(f)$ and $f^{\prime \prime}=\curvearrowright(f)$ then in $\Gamma \backslash e$ we set $\mathrm{mk}^{L}\left(f^{\prime}, f^{\prime \prime}\right)=\overline{\bar{a} \bar{b}}$.

\subsection{Spaces of graphs with metrics}

1.3.1. Graphs with a metric. A metric $w_{\Gamma}$ for a graph is a map $E_{\Gamma} \rightarrow \mathbb{R}_{>0}$. The (global) re-scaling of a metric $w$ by $\lambda$ is the metric $\lambda w,(\lambda w)(e)=\lambda(w(e))$. The length of a cycle $c$ is the sum of the lengths of its edges length $(c)=\sum_{f \in c} w(\{f, l(f)\})$. A metric for a treelike ribbon graph is called normalized if the length of each nondistinguished cycle is 1 . We will write $\mathcal{M} \mathcal{R} i b_{n, g}$ for the set of metric marked ribbon graphs of genus $g$ with $n$ boundary cycles.

1.3.2. Projective metrics. Notice that there is an $\mathbb{R}_{>0}$-action on $\mathcal{M} \mathcal{R} i b$ which scales the metric $\mu$ by an overall factor. This action of course preserves the genus and number of boundaries. We set $\mathbb{P} \mathcal{R} i b:=\mathcal{M} \mathcal{R} i b / \mathbb{R}>0$ using the same conventions 
as in Notation 1.3. The elements of $\mathbb{P} \mathcal{R} i b$ are called graphs with a projective metric. Notice that one can always choose a normalized representative for any projective metric. We set $\mathbb{P} R i b_{n, g}=\mathcal{M} \mathcal{R} i b_{n, g} / \mathbb{R}_{>0}$.

1.3.3. The space of metric ribbon graphs. We endow these above sets with a topology by constructing $\mathbb{P} \mathcal{R} i b(n, g)$ in the standard fashion. That is we realize them as a subspace of the quotient of the disjoint union of simplices by an equivalence relation. For each graph $\Gamma \in \mathcal{R} i b(n, g)$ with $|E(\Gamma)|=k+1$ we fix a $k$-simplex $\Delta_{\Gamma}$. Using barycentric coordinates for this simplex, a point of this simplex can be identified with a choice of projective weights on the edges. The points of $\mathbb{P} \mathcal{R} i b_{n, g}$ can thus be identified with the interior of the disjoint union over all $\Delta_{\Gamma}, \Gamma \in \mathcal{R i} b_{n, g}$. Furthermore the faces of $\Delta_{\Gamma}$ correspond to the edges of $\Gamma$. Now, we use the following identifications: A face of $\Delta_{\Gamma}$ is identified with $\Delta_{\Gamma / e}$ if $\Gamma / e \in \mathcal{R} i b_{n, g}$. We give the resulting space the quotient topology (this is actually a $\mathrm{CW}$ complex) and identify $\mathbb{P} \mathcal{R} i b$ with the image of the interiors of the $\Delta_{\Gamma}$. Then we give $\mathcal{M} \mathcal{R} i b:=\mathbb{P} \mathcal{R} i b \times \mathbb{R}_{>0}$ the product topology.

1.3.4. Marked ribbon graphs with metric and maps of circles. For a marked ribbon graph with a metric, let $c_{i}$ be its cycles, let $\left|c_{i}\right|$ be their image in the realization and let $r_{i}$ be the length of $c_{i}$. Then there are natural maps $\phi_{i}: S^{1} \rightarrow\left|c_{i}\right|$ which map $S^{1}$ onto the cycle by starting at the vertex $v_{i}:=\partial\left(\operatorname{mk}\left(c_{i}\right)\right)$ and going around the cycle mapping each point $\theta \in S^{1}$ to the point at distance $\frac{\theta}{2 \pi} r_{i}$ from $v_{i}$ along the cycle $c_{i}$. This observation connects the current constructions to those involving a

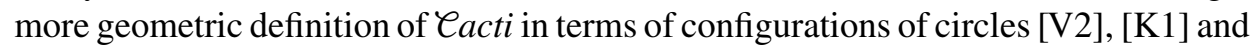
other geometric constructions involving such configurations such as the map Loop used for the $\mathcal{A} r c$ operad [KLP]. In particular the treelike ribbon graphs correspond to $C_{a c t i}$ and the spineless treelike ribbon graphs correspond to $C_{\text {act }}$.

\subsection{Di-graphs and Sullivan chord diagrams}

1.4.1. Ribbon di-graphs. A ribbon graph a di-graph is a ribbon graph $\Gamma$ together with a $\mathbb{Z} / 2 \mathbb{Z}$ labelling of the cycles of $\Gamma: i / o:\{$ cycles of $\Gamma\} \rightarrow \mathbb{Z} / 2 \mathbb{Z}$. We call the cycles $i / o^{-1}(0)=$ : Out $\Gamma$ the outgoing ones and $i / o^{-1}(1)=: \operatorname{In}_{\Gamma}$ the incoming ones. A di-graph is said to be of type $(n, m)$ if $\left|\operatorname{In}_{\Gamma}\right|=n$ and $\left|\mathrm{Out}_{\Gamma}\right|=m$. We will denote the set of these graphs by $\mathcal{R} i b^{i / o}$.

A ribbon di-graph is called perfectly partitioned if $i / o(l(f))=1-i / o(f)$ for every flag $f$. That is each edge is part of one input and one output cycle. We will call the set of these graphs $\mathcal{R} i b^{i \leftrightarrow o}$.

An $\left(S_{1}, S_{2}\right)$-labelled ribbon di-graph is a ribbon di-graph together with bijective maps In $\rightarrow S_{1}$ and Out $\rightarrow S_{2}$. We denote the induced map on In $\amalg$ Out by Lab. If $\left(S_{1}, S_{2}\right)$ is not mentioned, we will use $S_{1}=\bar{n}$ and $S_{2}=\bar{m}$ as the default indexing sets for a graph of type $(n, m)$. 
1.4.2. Sullivan chord and ribbon diagrams. There are many definitions of Sullivan chord diagrams in the literature [CS], [S1], [S2], [CG], [TZ]. We will use the following conventions.

Definition 1.4. A Sullivan chord diagram is a marked labelled ribbon di-graph which satisfies the following condition:

i) After deleting the edges of the incoming cycles one is left with a forest, i.e. a possibly disconnected set of contractible graphs.

We denote the set of these graphs by Chord ${ }^{\text {Sul }}$ and call them Sullivan chord diagrams.

A strict Sullivan chord diagram also satisfies the condition:

ii) It is possible to embed the realization of the graph into the plane in such a way that the images of the incoming cycles are disjointly embedded circles.

We denote this set of graphs by Chord ${ }^{\text {StSul }}$.

Remark 1.5. Notice that in a Sullivan chord diagram there are two types of edges, those which belong to the In cycles and those which belong to the tree part. These are also sometimes called ghost edges. The edges of the In cycles are traversed exactly once by the In cycles and once by the Out cycles and the edges. That is if a flag $f$ is an element of an In cycle, then $l(f)$ is an element of an Out cycle. The ghost edges are traversed in either direction by Out cycles, that is, both $f$ and $l(f)$ are elements of Out cycles.

If one contracts the ghost edges, one obtains a perfectly partitioned di-graph. For this reason perfectly partitioned di-graphs are sometimes called reduced Sullivan chord diagrams. Notice though that such a diagram is not a strict Sullivan chord diagram in the above definition, since the In cycles will share vertices.

1.4.3. Spaces of di-graphs. To each of these classes of graphs there is the corresponding space of graphs whose elements are the graphs of the given type together with a metric. The notation for these spaces of graphs which we will use is to write $\mathcal{M}$ in front of the symbol of the graphs, e.g. $\mathcal{M}$ Chord ${ }^{\mathrm{StSul}}$ and $\mathcal{M} \mathcal{R} i b^{i \leftrightarrow o}$. These spaces have a natural topology. First to each set of discrete data, such as the labelling and the di-graph labelling we associate a component, and then forgetting this extra data each of these components can be identified with a subspace of $\mathcal{M} \mathcal{R} i b$ defined by the underlying metric graphs.

It is clear that the spaces corresponding to $\mathcal{R} i b^{i \leftrightarrow o}$ and Chord ${ }^{\text {Sul }}$ are homotopy equivalent. The homotopy is given by changing the metric, by homogeneously scaling all the lengths of the ghost edges to zero.

We will not use strict Sullivan chord diagrams in this paper and we included them only to make contact with the literature where they do sometimes appear. The following important fact which one can show with some effort characterizes the weak homotopy type of strict Sullivan chord diagrams. 
Claim 1.6. The map $p: \mathcal{M}$ Chord ${ }^{\mathrm{StSul}} \rightarrow \mathcal{M} \mathcal{R} i b^{i \leftrightarrow o}$ which contracts all ghost edges is a weak fibration with contractible fibers. Hence $\mathcal{M} \mathcal{R} i b^{i \leftrightarrow o}$ and $\mathcal{M}$ Chord ${ }^{\mathrm{StSul}}$ are weakly homotopy equivalent.

\section{The Arc operad}

In this section, we start by giving a brief review of the salient features of the $\mathcal{A r c}$ operad of $[\mathrm{KLP}]$ which is reasonably self-contained. The presentation of the material closely follows the Appendix B of [K1]. For full details, we refer to [KLP]. In addition to this review, we furthermore introduce an equivalent combinatorial language which will be key for the following, in particular for [K4]. Simultaneously, we introduce new cell level structures and then go on to define new cell level operads and extensions of the A $r c$ operad structure.

2.1. Spaces of graphs on surfaces. Fix an oriented surface $F_{g, r}^{s}$ of genus $g$ with $s$ punctures and $r$ boundary components which are labelled from 0 to $r-1$, together with marked points on the boundary, one for each boundary component. We call this data $F$ for short if no confusion can arise.

The piece of the $\mathcal{A} r c$ operad supported on $F$ will be an open subspace of a space $\mathcal{A}_{g, r}^{s}$. The latter space is a CW complex whose cells are indexed by graphs on the surface $F_{g, r}^{s}$ up to the action of the pure mapping class group $P M C$ which is the group of orientation preserving homeomorphisms of $F_{g, r}^{s}$ modulo homotopies that pointwise fix the set which is the union of the set of the marked points on the boundary and the set of punctures. A quick review in terms of graphs is as follows.

2.1.1. Embedded graphs. By an embedding of a graph $\Gamma$ into a surface $F$, we mean an embedding $i:|\Gamma| \rightarrow F$ with the conditions:

i) $\Gamma$ has at least one edge.

ii) The vertices map bijectively to the marked points on the boundaries.

iii) No images of two edges are homotopic to each other, by homotopies fixing the endpoints.

iv) No image of an edge is homotopic to a part of the boundary, again by homotopies fixing the endpoints.

Two embeddings are equivalent if there is a homotopy of embeddings of the above type from one to the other. Note that such a homotopy is necessarily constant on the vertices.

The images of the edges are called arcs. And the set of connected components of $F \backslash i(\Gamma)$ are called complementary regions. 
Changing representatives in a class yields natural bijections of the sets of arcs and connected components of $F \backslash i(\Gamma)$ corresponding to the different representatives. We can therefore associate to each equivalence class of embeddings its sets of arcs together with their incidence conditions and connected components - strictly speaking of course the equivalence classes of these objects.

Definition 2.1. By a graph $\gamma$ on a surface we mean a triple $(F, \Gamma,[i])$ where $[i]$ is an equivalence class of embeddings of $\Gamma$ into that surface. We will denote the isomorphism class of complementary regions by $\operatorname{Comp}(\gamma)$. We will also set $|\gamma|=$ $\left|E_{\Gamma}\right|$. Fixing the surface $F$, we will call the set of graphs on a surface $\mathscr{E}(F)$.

2.1.2. A linear order on arcs. Notice that due to the orientation of the surface the graph inherits an induced linear order of all the flags at every vertex $F(v)$ from the embedding. Furthermore there is even a linear order on all flags by enumerating the flags first according to the boundary components on which their vertex lies and then according to the linear order at that vertex. This induces a linear order on all edges by enumerating the edges by the first appearance of a flag of that edge.

2.1.3. The poset structure. The set of such graphs on a fixed surface $F$ is a poset. The partial order is given by calling $\left(F, \Gamma^{\prime},\left[i^{\prime}\right]\right) \prec(F, \Gamma,[i])$ if $\Gamma^{\prime}$ is a subgraph of $\Gamma$ with the same vertices and $\left[i^{\prime}\right]$ is the restriction of $[i]$ to $\Gamma^{\prime}$. In other words, the first graph is obtained from the second by deleting some arcs.

We associate a simplex $\Delta(F, \Gamma,[i])$ to each such graph. $\Delta$ is the simplex whose vertices are given by the set of arcs/edges enumerated in their linear order. The face maps are then given by deleting the respective arcs. This allows us to construct a CW complex out of this poset.

Definition 2.2. Fix $F=F_{g, r}^{s}$. The space $\mathcal{A}_{g, r}^{\prime s}$ is the space obtained by gluing the simplices $\Delta\left(F, \Gamma^{\prime},\left[i^{\prime}\right]\right)$ for all graphs on the surface according to the face maps.

The pure mapping class group naturally acts on $\mathcal{A}_{g, r}^{\prime s}$ and has finite isotropy [KLP].

Definition 2.3. The space $\mathcal{A}_{g, r}^{s}:=\mathcal{A}_{g, r}^{s} / P M C$.

\subsubsection{CW structure of $\mathcal{A}_{g, r}^{s}$}

Definition 2.4. Given a graph on a surface, we call its $P M C$ orbit its arc graph. If $\gamma$ is a graph on a surface, we denote by $\bar{\gamma}$ its arc graph or $P M C$ orbit. We denote the set of all arc graphs of a fixed surface $F$ by $\overline{\mathscr{E}}(F)$. A graph is called exhaustive if there are no vertices $v$ with $\operatorname{val}(v)=0$. This condition is invariant under $P M C$ and hence we can speak about exhaustive arc graphs. The set of all exhaustive arc graphs on $F$ is denoted by $\overline{\mathscr{g}}^{e}(F)$. 
Notice that since the incidence conditions are preserved, we can set $|\bar{\gamma}|=|\gamma|$ where $\gamma$ is any representative and likewise define $\operatorname{Comp}(\bar{\gamma})$. We call an arc graph exhaustive if and only if it contains no isolated vertices, that is vertices with $\operatorname{val}(v)=0$.

Now by construction it is clear that $\mathcal{A}_{g, r}^{s}$ is realized as a CW complex which has one cell for each arc graph $\bar{\gamma}$ of dimension $|\gamma|-1$. Moreover the cell for a given class of graphs is actually a map of a simplex whose vertices correspond to the arcs in the order discussed above. The attaching maps are given by deleting edges and identifying the resulting face with its image. Due to the action of $P M C$ some of the faces of might become identified by these maps, so that the image will not necessarily be a simplex. The open part of the cell will however be an open simplex. Let $C(\bar{\alpha})$ be the image of the cell and $\dot{C}(\bar{\alpha})$ be its interior, then

$$
\mathcal{A}_{g, r}^{s}=\bigcup_{\bar{\alpha} \in \overline{\mathscr{E}}\left(F_{g, r}^{s}\right)} C(\bar{\alpha}), \quad \mathcal{A}_{g, r}^{s}=\bigsqcup_{\bar{\alpha} \in \overline{\mathscr{E}}\left(F_{g, r}^{s}\right)} \dot{C}(\bar{\alpha})
$$

Let $\Delta^{n}$ denote the standard $n$-simplex and $\dot{\Delta}$ its interior then $\dot{C}(\gamma)=\mathbb{R}_{>0}^{\left|E_{\Gamma}\right|} / \mathbb{R}_{>0}=$ $\dot{\Delta}^{\left|E_{\Gamma}\right|-1}=: C(\Gamma)$ which only depends on the underlying graph $\Gamma$ of $\gamma$.

This also means that the space $\mathcal{A}_{g, r}^{s}$ is filtered by the cells of dimension less than or equal to $k$. We will use the notation $\left(\mathcal{A}_{g, r}^{s}\right) \leq k$ for the pieces of this filtration.

2.1.5. Elements of the $\mathcal{A}_{g, r}^{s}$ as projectively weighted graphs. Using barycentric coordinates for the open part of the cells the elements of $\mathcal{A}_{g, r}^{s}$ are given by specifying an arc graph together with a map $w$ from the edges of the graph $E_{\Gamma}$ to $\mathbb{R}_{>0}$ assigning a weight to each edge s.t. the sum of all weights is 1 .

Alternatively, we can regard the map $w: E_{\Gamma} \rightarrow \mathbb{R}_{>0}$ as an equivalence class under the equivalence relation of, i.e., $w \sim w^{\prime}$ if there exists $\lambda \in \mathbb{R}_{>0}$ such that $w(e)=\lambda w^{\prime}(e)$ for all $e \in E_{\Gamma}$. That is $w$ is a projective metric. We call the set of $w(e)$ the projective weights of the edges. In the limit, when the projective weight of an edge goes to zero, the edge/arc is deleted, see [KLP] for more details. For an example see Figure 1, which is discussed below.

An element $\alpha \in \mathcal{A}_{g, r}^{s}$ can be described by a tuple $\alpha=(F, \Gamma, \overline{[i]}, w)$ where $F$ and $\Gamma$ are as above, $\overline{[i]}$ is a PMC orbit of an equivalence class of embeddings and $w$ is a projective metric for $\Gamma$. Alternatively it can be described by a tuple $(\bar{\gamma}, w)$ where $\bar{\gamma} \in \overline{\mathscr{E}}(F)$ and $w$ is a projective metric for the underlying abstract graph $\Gamma$.

Example 2.5. $\mathcal{A}_{0,2}^{0}=S^{1}$. Up to $P M C$ there is a unique graph with one edge and a unique graph with two edges. The former gives a zero-cell and the latter gives a onecell whose source is a 1-simplex. Its two subgraphs with one edge that correspond to the boundary lie in the same orbit of the action of $P M C$ and thus are identified to yield $S^{1}$. The fundamental cycle is given by $\Delta$ of Figure 1 . 

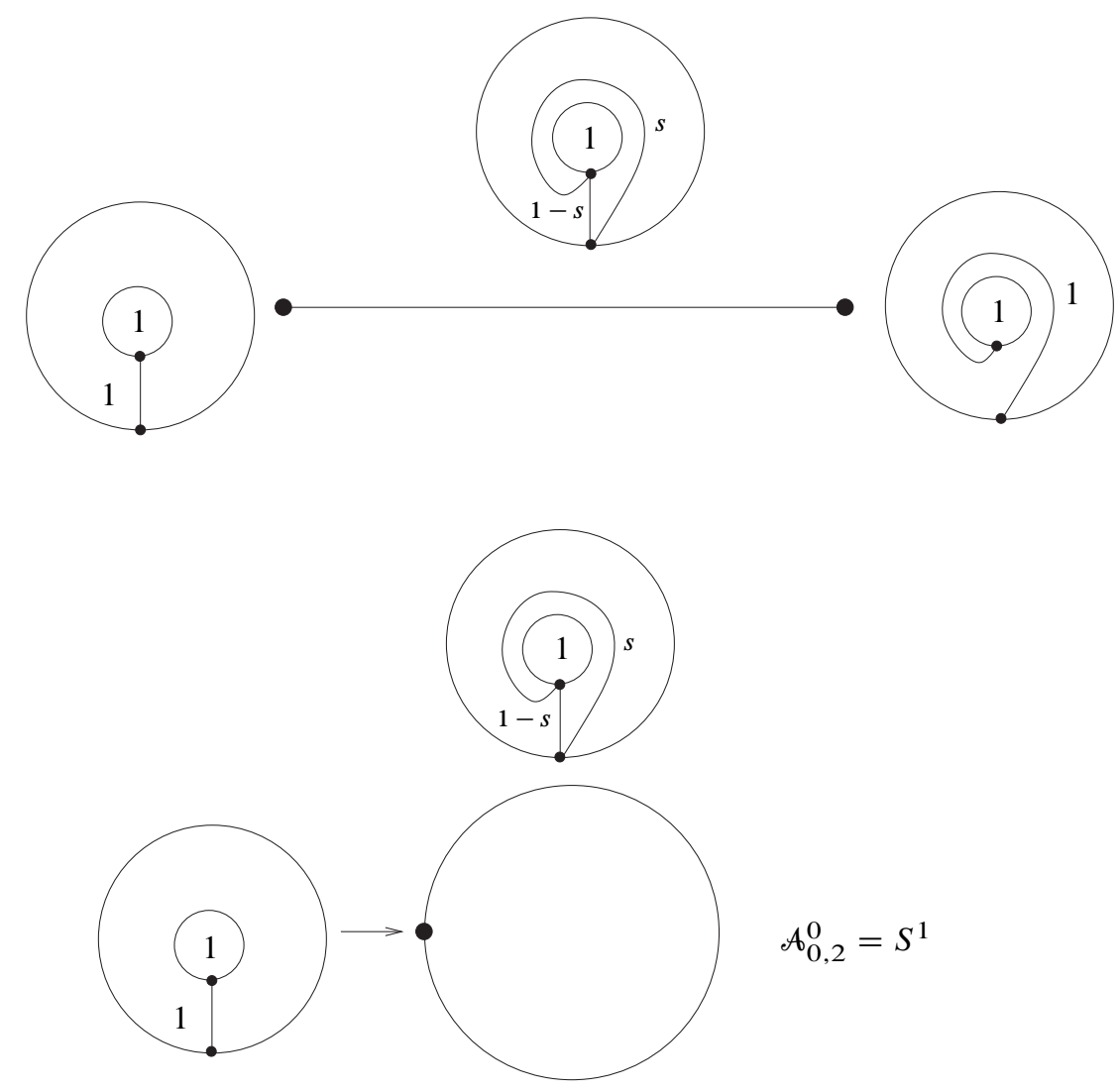

Figure 1. The space $\mathcal{A}_{0,2}^{0}$ is given as the $\mathrm{CW}$ decomposition of $S^{1}$ with one 0 -cell and one 1-cell. It can be thought of as the quotient of the interval in which the endpoints are identified by the action of the pure mapping class group. The generator of $C C_{*}\left(S^{1}\right)$ is called $\Delta$.

2.1.6. Drawing pictures for arcs. There are several pictures one can use to view elements of $\mathcal{A}$. In order to draw elements them it is useful to expand the marked point on the boundary to an interval called window, and let the arcs end on this interval according to the linear order. Equivalently, one can mark one point of the boundary and let the arcs end in their linear order anywhere but on this point. We will mostly depict arc graphs in the latter manner. See Figure 2 for an example of an arc graph - all arcs running to the marked points - and its alternate depiction with none of the arcs hitting the marked point and all arcs having disjoint endpoints.

Notation 2.6. Since in the following we will always be dealing with arc graphs, we will now omit the over-line in the notation. Hence we will write $\gamma \in \overline{\mathscr{E}}(F)$. We also 

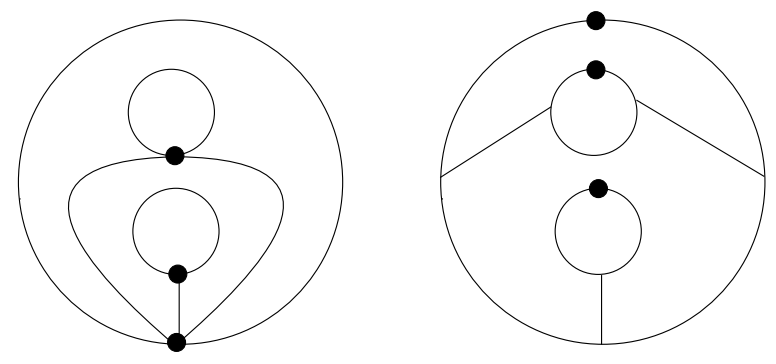

Figure 2. An arc graph and its alternate depiction with disjoint arcs not hitting the marked points on the boundary.

fix that $\Gamma(\gamma)$ is the underlying graph. Furthermore elements of $\mathcal{A}_{g, r}^{s}$ will usually be called $\alpha$ and $\beta$. If $\alpha \in \mathcal{A}_{g, r}^{s}$ we fix that $\gamma(\alpha), \Gamma(\alpha)$ and $w(\alpha)$ are the underlying arc graph, its underlying graph and the projective metric, respectively.

Definition 2.7. We define the Euler characteristic of an element $\alpha \in A_{g, r}^{s}$ to be $\chi(\alpha)=|\operatorname{Comp}(\alpha)|-|E(\Gamma(\alpha))|$.

Lemma 2.8. The following inequality holds

$$
\chi(\alpha) \geq \chi(F(\alpha)),
$$

and the equality holds if and only if the complementary regions are polygons.

Proof. If the complementary regions are polygons, we obtain a triangulation of the surface and hence $\chi(F)=\left|V_{\Gamma}\right|-\left|E_{\Gamma}\right|-r+|\operatorname{Comp}(\alpha)|$. There are $\left|V_{\Gamma}\right|=r$ vertices and $\left|E_{\Gamma}\right|+r$ edges since the boundaries also count as edges. In this count, the complementary region contributes 1 , as it should for a polygon. If the complementary regions have some topology then their Euler characteristic is strictly less than 1 and the Euler characteristic of the surface would be smaller; whence the claim.

\subsection{Topological operad structure}

2.2.1. The spaces $\operatorname{Arc}(\boldsymbol{n})$. We begin by reviewing the construction of [KLP]. We then recast it into a purely combinatorial way. This will allow us to define the actions of [K4] more simply, but also allow us to show that although $\mathcal{A r} c_{\#}$ is not an operad on the topological level, it is a rational operad and gives rise to a cellular operad.

Definition 2.9. We define $\mathcal{A} r c_{g}^{s}(n) \subset \mathcal{A}_{g, n+1}^{s}$ to be the subset of those weighted arc graphs whose arc graph is exhaustive. We define $\operatorname{Arc}(n):=\coprod_{s, g \in \mathbb{N}} \mathcal{A}_{r} c_{g}^{s}(n) .^{2}$

\footnotetext{
${ }^{2}$ Unfortunately there is a typo in the definition of $\mathcal{A} r c(n)$ in $[\mathrm{KLP}]$ where $\amalg$ was inadvertently replaced by the direct limit.
} 
2.2.2. Open-cell cell complex. It is clear by construction that the A $r$ rc operad again has a decomposition into open cells,

$$
\mathcal{A} r c_{g}^{s}(n)=\bigsqcup_{\gamma=\bar{g} e}\left(F_{g, n+1}^{s}\right) \dot{C}(\gamma) .
$$

Again $\dot{C}(\gamma)=\mathbb{R}_{>0}^{\left|E_{\Gamma}\right|} / \mathbb{R}_{>0}=\dot{\Delta}^{\left|E_{\Gamma}\right|-1}:=\dot{C}(\Gamma)$ only depends on the underlying graph $\Gamma$ of $\gamma$.

We will denote the free Abelian group generated by the $C(\alpha)$ as above by $\ell_{o}^{*}(\mathcal{A} r c)_{g}^{s}(n)$. We will write $\bigodot_{o}^{*}(\mathcal{A} r c)(n)=\bigsqcup_{g, s} \bigodot_{o}^{*}(\mathcal{A} r c)_{g}^{s}(n)$ and $\bigodot_{o}^{*}(\mathcal{A} r c)=$ $\bigsqcup_{n} \mathcal{C}_{o}^{*}(\mathcal{A} r c)(n)$. We choose the notation to reflect the fact that we are strictly speaking not dealing with cellular chains, however see \$3.4.1.

$\mathcal{C}_{o}^{*}(\mathcal{A} r C)(n)$ is also graded by the dimension of the cells, we will write $\mathcal{C}_{o}^{*}(\mathcal{A} r c)(n)^{k}$ for the subgroup generated by cells of dimension $k$ and we will also write $\mathcal{C}_{o}^{*}(\mathcal{A} r c)(n)^{\leq k}$ for the subgroup of cells of dimension $\leq k$. It is clear that $\mathcal{C}_{o}^{*}(\mathcal{A} r c)(n) \leq k$ induces a filtration on $\mathcal{C}_{o}^{*}(\mathcal{A} r c)(n)$ and that the associated graded is isomorphic to the direct sum of the $\mathcal{C}_{o}^{*}(\mathcal{A} r c)(n)^{k}$

$$
\operatorname{Gr} \ell_{o}^{*}(\mathcal{A} r c):=\operatorname{Gr}\left(\bigodot_{o}^{*}(\mathcal{A} r c)(n), \leq\right) \simeq \bigoplus_{k} \bigodot_{o}^{*}(\mathcal{A} r c)^{k}(n)
$$

The differential $\partial$ of $\mathcal{A}_{g, r}^{s}$ also descends to $\bigodot_{o}^{*}(\mathcal{A} r c)$ and $\operatorname{Gr} \bigodot_{o}^{*}(\mathcal{A} r c)$ by simply omitting the cells which are not in A $r$ c. Applying the differential twice will kill two arcs, each original summand will either be twice treated as zero or appear with opposite sign as in $\mathcal{A}_{g, r}^{s}$. Hence the differential squares to zero.

2.2.3. Relative cells. The complex $\mathcal{C}_{o}^{*}(\mathcal{A} r c)_{g}^{s}(n)$ and the isomorphic complex $\operatorname{Gr} \mathcal{C}_{o}^{*}(\mathcal{A} r c)_{G}^{s}(n)$ can be identified with the complex of relative cells $C C_{*}(A, A \backslash \mathcal{A} r c)$.

Notice that the spaces $\mathcal{A} r c(n)$ have a natural operations of $\Phi_{n}$ which permutes the labels $\{1, \ldots, n\}$ and one of $\mathbb{S}_{n+1}$ which permutes the labels $\{0, \ldots, n\}$. Also notice that the spaces $\mathcal{A} r c_{g}^{s}(n)$ inherit the grading and filtration from $\mathcal{A}_{g}^{s}(n)$. This is also true for their unions $\mathcal{A} r c(n)$ and we will write $\mathcal{A} r c(n)^{\leq k}$ for these pieces. That is if $\alpha \in \mathcal{A} \operatorname{rrc}(n)^{\leq k}$ then $|E(\Gamma(\alpha))| \leq k+1$.

2.2.4. Topological description of the gluing [KLP]. To give the composite $\alpha \circ_{i} \alpha^{\prime}$ for two arc families $\alpha=(F, \Gamma, \overline{[i]}, w) \in \mathcal{A} r c(m)$ and $\alpha^{\prime}=\left(F^{\prime}, \Gamma^{\prime}, \overline{\left[i^{\prime}\right]}, w^{\prime}\right) \in$ $\operatorname{Arc}(n)$ one most conveniently chooses metrics on $F$ and $F^{\prime}$. The construction does not depend on the choice. With this metric, one produces a partially measured foliation in which the arcs are replaced by bands of parallel leaves (parallel to the original arc) of width given by the weight of the arc. For this we choose the window representation and also make the window tight in the sense that there is no space between the bands and between the end-points of the window and the bands. Finally, we put in the separatrices. The normalization we choose is that the sum of the weights at boundary $i$ 
of $\alpha$ coincides with the sum of the weights at the boundary 0 , we can also fix them both to be one. Now when gluing the boundaries, we match up the windows, which have the same width, and then just glue the foliations. This basically means that we glue two leaves of the two foliations if they end on the same point. We then delete the separatrices. Afterwards, we collect together all parallel leaves into one band. In this procedure, some of the original bands might be split or "cut" by the separatrices. We assign to each band one arc with weight given by the width of the consolidated band. If arcs occur, which do not hit the boundaries, then we simply delete these arcs. We call these arcs or bands "closed loops" and say that "closed loops appear in the gluing".

Notice that after gluing there will be no parallel arcs, since all parallel leaves are collected into one band and the condition of being parallel is PMC invariant - before and after gluing.

Theorem 2.10 ([KLP]). Together with the gluing operations above, the spaces Arc form a cyclic operad.

Lemma 2.11. The gluing operations are compatible with the filtrations, i.e., $\operatorname{Arc}(m) \leq p \circ_{i} \operatorname{Arc}(n)^{\leq q} \subset \operatorname{Arcc}(n+m-1) \leq p+q$.

Proof. If $k$ is the number of arcs at the boundary $i$ of $\alpha$ and $l$ is the number of arcs at the boundary 0 of $\beta$ then the number of glued arcs resulting from these arcs via the operadic composition $\circ_{i}$ is at most $k+l-1$. This comes from the fact that each "cut" (that is each separatrix) of the glued foliation contains at least one of the separatrices of the two foliations before gluing. Hence the number of $\operatorname{arcs}$ of $\alpha \circ_{i} \beta$ is at most $p+1-k+q+1-l+k+l-1=p+q+1$, and the claim follows.

2.2.5. Combinatorial description. One can also give a purely combinatorial description of the gluing in which we define the new graph on the glued surface. An example of a gluing is given in Figure 3.

In the first step we normalize as above to make the sums of weights match. The weights of the arcs incident to the boundaries 0 and $i$ then define two partitions $P_{0}$ and $P_{i}$ of the unit interval where each flag incident to the vertices 0 and $i$ corresponds now to a subinterval of length given by the weight of the respective arc.

Let us first also assume that there are no arcs running from 0 to 0 or from $i$ to $i$. We will call such arcs recursive. Hence we are for the moment assuming that there are no recursive arcs. Let $\mathcal{P}_{\circ}$ be the biggest common sub-partition. If in the common partition $\mathcal{P}_{\circ}$ a subinterval $I^{\prime}$ of $P_{0}$ or $P_{i}$ is subdivided, let $f$ be the flag associated to $I^{\prime}$ and duplicate the edge $e=\{f, l(f)\}$ so that there are now two copies $e^{(1)}$ and $e^{(2)}$ of $e$ which are embedded to be parallel. Associate the copies $f^{(1)}$ and $f^{(2)}$ of

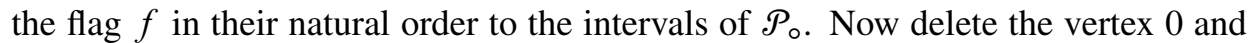
all flags of the vertex 0 of $\alpha^{\prime}$ and the vertex $i$ and all the flags of the vertex $i$ of $\alpha$. 

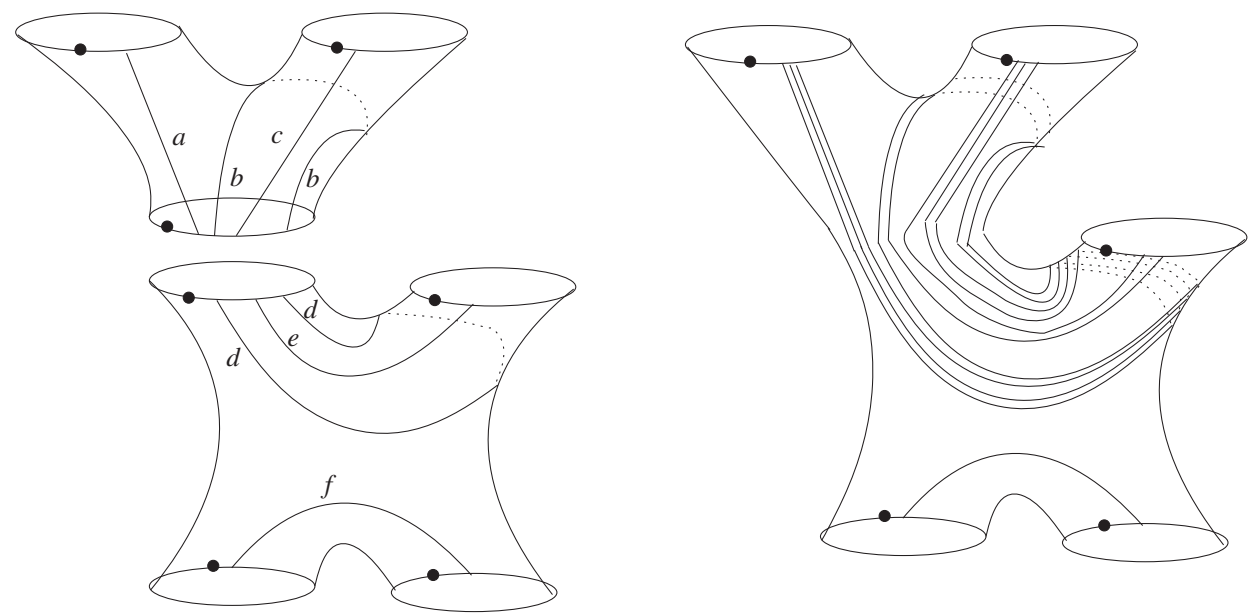

a)

b)
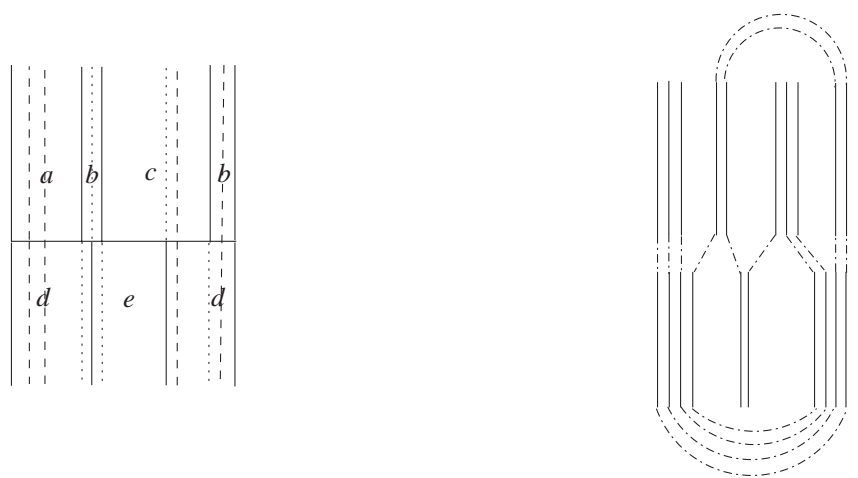

c)

d)

Figure 3. a) The arc graphs which are to be glued assuming the relative weights $a, b, c, d$ and $e$ as indicated by the solid lines in c). b) The result of the gluing (the weights are according to $c$ ). c) The combinatorics of cutting the bands. The solid lines are the original boundaries the dotted lines are the first cuts and the dashed lines represent the recursive cuts. d) The combinatorics of splitting, and joining flags.

Then glue the remaining graphs with the duplicated flags by defining the involution $l_{\circ}$ on the flags of this graph by extending the involution induced by the two original arc graphs, by setting $u=l_{\circ}(d)$ if $l(u)$ and $l(d)$ correspond to the same subinterval $I^{\prime \prime}$ of the partition $\mathcal{P}_{0}$. The weight of such a glued edge is defined to be the length of the subinterval $I^{\prime \prime}$. 
In the case that there are recursive arcs the combinatorics are a little more difficult. We start out as above and will call a biggest common partition $\mathcal{P}$. A subinterval is called recursive if it belongs to a flag which corresponds to a recursive arc. Now, we have to complete the partitions. This means that if a subinterval is recursive, we have to partition the interval corresponding to $l(f)$ in the induced orientation just as the interval corresponding to $f$. This amounts to cutting the band corresponding to the edge $(f, l(f))$ according to the partition. One might have to iterate this process. The process will stabilize however, since there are only finitely many intervals and cuts; and for that matter only finitely many bands after gluing [KLP]. This will yield a partition $\mathcal{P}_{\circ}$ of the interval. We now proceed with the combinatorics as above. Thus by replicating edges, we obtain two flags per subinterval of $\mathcal{P}_{\circ}$, one on each side. The glued graph is now defined in three steps. First fix one vertex for each subinterval an define the two flags incident to the subinterval to be incident to the vertex. Secondly, delete these vertices of valence two and their flags. Thirdly glue the remaining unpaired flags to edges using $l_{0}$ which is defined analogously as above.

For the embedding one again uses the window description. After possible duplications the arcs can be arranged to end in the mid-point of the subintervals of $P_{\circ}$ and the embedding is defined by connecting two arcs if their endpoints coincide.

In both cases, there is a last stabilization step if the edges corresponding to the first and last interval are parallely embedded. If this occurs, merge the edges together to one edge and define the weight of this edge to be the sum of the weights of the parallel edges.

Notice that this prescription automatically deletes any closed arcs, viz. bands not hitting the boundaries that might appear in the second step.

Notation 2.12. We wish to fix the following terminology. In the gluing of two families there are two possibilities for a non-recursive arc which is glued. It is either split if the flag not incident to the boundary on which the gluing is performed is duplicated or not split if the opposite is the case. If the flag is duplicated, it gives rise to a new angle in the glued graph which we call a split angle.

We also call an arc family twisted at the boundary $i$ if the first and the last arcs incident to the boundary $i$ become parallel if one allows homotopies moving the endpoints around the boundary $i$. We use the same terminology for the underlying graphs.

2.2.6. Combinatorics of the gluing. For future reference, we wish to detail the combinatorics of the gluing if the weights on the arcs on both the boundaries which are to be glued vary freely.

First we treat the case where there are no arcs running from 0 to 0 or from $i$ to $i$. Also for the moment assume not both boundaries are twisted. Fix $\alpha \in \mathcal{A} r c(n)$ and $\beta \in \mathcal{A} r c(m)$ and then let $\alpha$ and $\beta$ vary freely inside their cell. Assume that inside the cell $\alpha$ has $k$ arcs with freely varying weights on the boundary $i$ and $\beta$ has $l$ arcs 
with freely varying weights on the boundary 0 . Then the gluing will generically glue these $k+l$ arcs into $k+l-1$ arcs, since the generic common partition will have exactly that many components. The combinatorics are hence the decomposition of $\Delta^{k-1} \times \Delta^{l-1}=\sum_{\sigma \in \operatorname{Sh}(k, l)} \Delta^{k+l-2}$ where $\operatorname{Sh}(k, l)$ are the shuffles of type $k, l$. A non-generic situation happens if some of the partition points match up this corresponds passing to the faces of the simplices. This set is at least of codimension one.

If both gluing boundaries are twisted, we generically only obtain $k+l-2 \operatorname{arcs}$ since the two outside arcs will always be parallel after gluing.

Now say that there are $s$ arcs running from 0 to 0 and $t$ arcs from $i$ to $i$. Let $k$ and $l$ be as above. We notice that the number of arcs after gluing is again generically $k+l-1$ arcs. For this, we notice that the final number of arcs is given by the half the number of replicated flags for the gluing minus the number of flags which run from the boundary back to itself. Or in the notation of $\$ 2.2 .6$, the number of subintervals of $\mathcal{P}_{\circ}$ minus half the number of recursive flags. So we are left with one half the number of the non-recursive flags. The number of these flags corresponds to the interval markings or cuts on the respective subintervals. Now counting only these markings, we count each original cut (subinterval of $\mathcal{P}$ before the iteration process of §2.2.6) exactly once, since the iteration stops exactly if the opposite side of the interval is non-recursive. The number of these cuts is $k+l$ as above. The indexing of the occurring combinatorics can again be done by $\operatorname{Sh}(k, l)$ as per the original cuts, viz. $\mathcal{P}$, but the topology of the resulting arcs is more complex. Now there are two types of non-generic situations. The first is as above, that is the situation corresponding to the partitioning points matching up. This is again of codimension one. The second is given by bands which form closed loops that are erased. This is actually even of codimension two, since in order for a closed loop to form two of the cuts have to align.

2.2.7. Partial operad structure on $\mathcal{A}_{g, r}^{s}$. We wish to point out that the gluing we defined in [KLP] that is reviewed above extends to a partial cyclic operad structure on $\mathcal{A}(n):=\bigsqcup_{s, g} A_{g, n+1}^{s}$. The $\mathbb{S}_{n+1}$-action is taken to be the one permuting the labels. The gluings $o_{i}$ above are well defined as soon as there are arcs incident to both $i$ and 0 on the surfaces which are glued along these boundaries.

\subsection{Operad structures on the cell level}

2.3.1. Singular chains/PL chains. First it is clear that there are operations which have as input the singular co-chains of the $\mathcal{A} r c$ operad and as output products of these chains. So that if we move to PL-chains, we indeed get an operad action on the chain level. This is the point of view taken in [KLP] where we showed that this cell level operad has a Gerstenhaber-Batalin-Vilkovisky (GBV) structure up to explicitly given homotopies. 
2.3.2. Operad structures on the free Abelian group of open cells. There are two operad structures on the open/relative cell level. The first is the more naïve induced operad structure on the open cells. And the second one is its associated graded which is more suited for the description of moduli space.

To give the first operad structure we claim that as sets

$$
\dot{C}(\alpha){ }_{k} \dot{C}(\beta)=\bigsqcup_{i \in I} \dot{C}\left(\gamma_{i}\right)
$$

for some index set $I$. The reason for this is that if a point of a cell $C(\gamma)$ is in the image, the whole cell is. It is possible to obtain all weights by varying the weights of the $\alpha$ and $\beta$ accordingly, see $\$ 2.2 .6$ and Lemma 2.13 below. By Lemma 2.11 the dimension of the cells $C\left(\gamma_{i}\right)$ appearing in the image is less or equal to the sum of the dimensions $\dot{C}(\alpha)$ and $\dot{C}(\beta)$. Finally, again by $\S 2.2 .6$ and Lemma 2.13, we have cells of the top-possible dimension if and only if not both the boundaries on which one glues are twisted. Lastly, the gluing map between cells is also 1-1 precisely when there is no twisting at both boundaries and there are no closed loops.

Lemma 2.13. The map $\circ_{i}$ restricted to $\dot{C}(\alpha) \times \dot{C}(\beta)$ is a bijection onto its image if and only if not both $\alpha$ is twisted at the boundary $i$ and $\beta$ is twisted at the boundary 0 and there are no closed loops. In the case not both boundaries are twisted $\circ_{i}$ restricted to the set of metric arc graphs for which no closed loops appear is bijective onto is image.

Proof. Fix two surfaces $F_{g, r}^{s}$ and $F_{g^{\prime}, r^{\prime}}^{\prime s^{\prime}}$ and let $F^{\prime \prime}=F_{g+g^{\prime}, s+s^{\prime}}^{\prime \prime s+s^{\prime}}$ be the surface obtained from $F$ and $F^{\prime}$ by gluing the boundary 0 of $F^{\prime}$ to the boundary $i$ of $F$ and let $l$ the simple closed curve which is the image of the boundaries $i$ and 0 in the glued surface. To avoid too many super- and subscripts set $\operatorname{Arc}(F):=\operatorname{Arc}\left(F_{g, r}^{s}\right):=\mathcal{A}_{g, r}^{s}$ and likewise for $F^{\prime}$ and $F^{\prime \prime}$. Assuming that there are no closed loops, the gluing procedure is such, that the preimage of $o_{i}$ from $\operatorname{Arc}(F) \times \operatorname{Arc}\left(F^{\prime}\right) \rightarrow \operatorname{Arcc}\left(F^{\prime \prime}\right)$ can be described as follows. Given a weighted arc graph $\gamma$ on the glued surface $F^{\prime \prime}$ all the preimages that is $\alpha, \beta$ such that $\alpha \circ_{i} \beta=\gamma$ are obtained by first fixing a point $p$ on the curve $l$, then cutting the surface $F^{\prime \prime}$ along $l$ and finally merging all the parallel bands which might occur after cutting while summing their weights. Here we allow the point $p$ to "split an arc". By this we mean that given a fixed arc with weight $w$ we draw a parallel arc to this arc (say to the right of the arc) and choose $w_{1}, w_{2}$ with $w_{1}+w_{2}=w$ and put a point between the two parallel arcs, that is to the right of the original arc. Fixing the cells $\dot{C}(\alpha)$ and $\dot{C}(\beta)$ we see that the choice of the point on $l$ is fixed up to moving the point along $l$ but not crossing any arc. From this description it follows that $o_{i}$ is injective precisely if this point $p$ does not "split an arc". In the case of closed loops, we see that we cannot detect them on the glued side and that accordingly, the parameters given by their width are free parameters in the pre-image. 
We will accordingly split the indexing set $I$ of equation 2.5 into $I^{\prime}$ which indexes the cells that are in the image of the gluings which do not exhibit any closed loops and $I^{\prime \prime}$ which indexes the cells whose graphs are obtained by erasing closed loops. As sets, we have:

$$
\dot{C}(\alpha) \circ_{k} \dot{C}(\beta)=\left(\coprod_{i \in I^{\prime}} \dot{C}\left(\gamma_{i}^{\prime}\right)\right) \amalg\left(\coprod_{i^{\prime \prime} \in I^{\prime \prime}} \dot{C}\left(\gamma_{i}^{\prime}\right)\right) .
$$

We define

$$
\dot{C}(\alpha) \tilde{\circ}_{k} \dot{C}(\beta):= \begin{cases}\sum_{i^{\prime} \in I^{\prime}} \pm \dot{C}\left(\gamma_{i^{\prime}}\right) & \text { if } \text { not both } \alpha \text { is twisted at the boundary } k \\ 0 & \text { and } \beta \text { is twisted at the boundary } 0, \\ & \text { if both } \alpha \text { is twisted at the boundary } k \\ & \text { and } \beta \text { is twisted at the boundary } 0\end{cases}
$$

in $\mathcal{C}_{o}^{*}(\mathcal{A} r c)(n)$ where the sign comes from the orientation of the cells given by the enumeration of the arcs.

Lemma 2.14. With the induced operad structure $\mathcal{C}_{o}^{*}(\mathcal{A} r c)$ is a cyclic operad which respects the filtration by dimension.

Proof. The fact that the $\mathbb{S}_{n}$-actions permuting the labels together with the operations defined in equation (2.7) yield an operad structure follows from the observations of the previous paragraph summed up $\S 2.2 .6$. The cyclicity under the $\mathbb{\$}_{n+1}$ action is inherent. The last statement follows from Lemma 2.11.

Corollary 2.15. The set of associated graded spaces $\mathrm{Gr} \mathcal{C}_{o}^{*}(\mathcal{A} r c)$ together with the action of the permutation groups on the labels and the operations induced from equation (2.7) form a cyclic operad.

Remark 2.16. Both operad structures are not structures of dg operads, for the following simple reason. If we glue together two surfaces then on the glued side, the limit in which the weight of all the arcs hitting the separating curve which is the image of the two glued boundaries goes to zero is possibly allowed and possibly contributes to the boundary of the cell. This limit, however, is not allowed for the two components, i.e. the result of this limit does not lie in $\partial \dot{C}(\alpha) \circ_{i} \dot{\beta} \cup \dot{C}(\alpha) \circ_{i} \partial \dot{\beta}$. For an example, see Figure 4 . Here the two families have trivial boundary individually in $\bigcup_{o}^{*}(\mathcal{A} r c)$, but their composition has a non-trivial boundary, since the limit where $a$ tends to zero is well defined in $\mathcal{A} r$ r.

We wish to point out that this limit is not allowed in $\mathcal{A} r_{\# \#}$, see Section §2.4. On the cellular level there are ways to remedy this situation, see \$3.1.1.

Let us make the cell level operation on the associated graded explicit. If $C(\alpha) \in$ $\mathcal{A} r c^{k}(n)$ and $C(\beta) \in \mathcal{A} r c^{l}(m)$ we obtain

$$
\dot{C}(\alpha) \tilde{\circ}_{k} \dot{C}(\beta)=\sum_{i \in I} \pm \dot{C}\left(\gamma_{i}\right)+\sum_{j \in J} \pm C\left(\delta_{j}\right)
$$



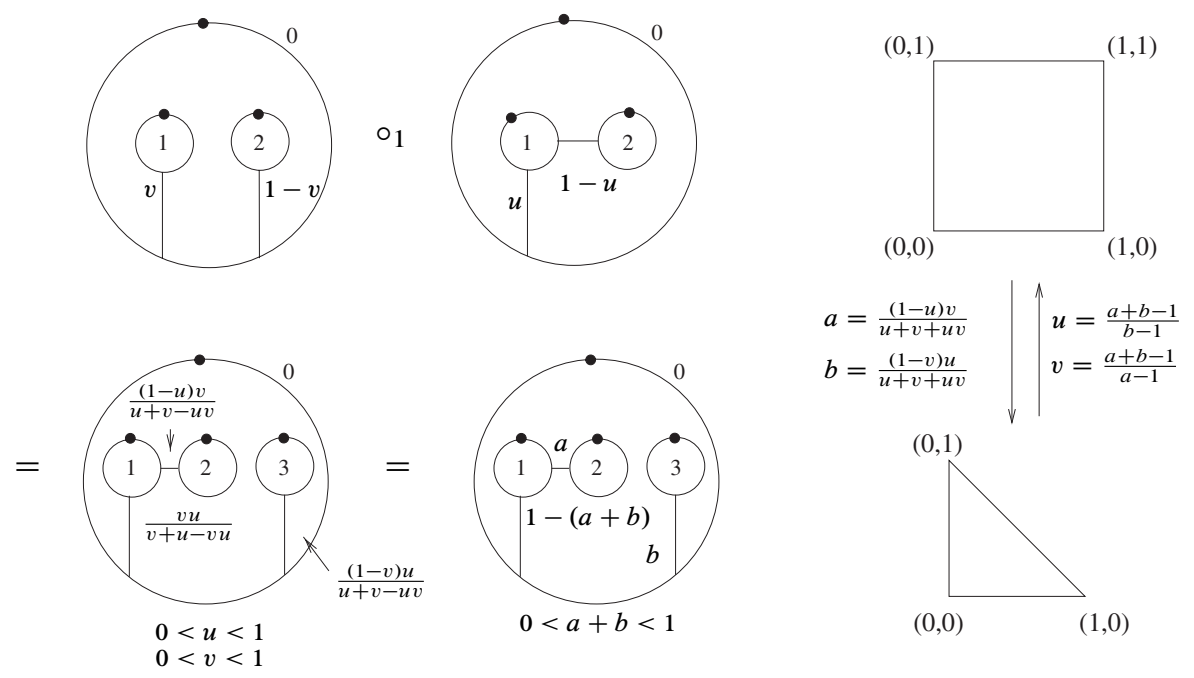

Figure 4. A gluing in $\mathcal{C}_{o}^{*}(\mathcal{A} r c)$ together with a reparameterization of $(0,1) \times(0,1)$ to the inside of the 2-simplex.

with $C\left(\gamma_{i}\right) \in \mathcal{A} r c^{k+l}(n+m)$ and $\operatorname{Cyc}\left(\delta_{j}\right) \in \mathcal{A} r c^{<k+l}(n+m)$ for some index sets $I$ and $J$.

Therefore, we get an induced operad structure on $\mathrm{Gr}^{*}(\mathcal{A} r c)$ by setting

$$
\dot{C}(\alpha){ }^{\circ} \dot{C}(\beta)=\sum_{i \in I} \pm C\left(\gamma_{i}\right)
$$

where \pm is the usual sign corresponding to the orientation, which is obtained from the shuffle of the edges that is induced by the respective shuffle in the product of sub-simplices $\Delta^{k-1} \times \Delta^{l-1}$ as discussed in $\$ 2.2 .6$.

The result of the operation (2.8) is zero exactly if the index set $I$ is empty and this is the case if and only if both $\alpha$ is twisted at $i$ and $\beta$ is twisted at 0 . Otherwise $I$ coincides with the set $\operatorname{Sh}(k, l)$ of $(k, l)$-shuffles.

An example of the gluing is given in Figure 5. Here the "diagonal" family is of codimension 1 and is included in the open cell gluing while in the graded gluing it is set to zero.

2.3.3. Relative cells. Another way to phrase the graded construction of the last paragraph is that we have defined an operad structures on the relative cells $C C_{*}(A, A \backslash \mathcal{A} r c)$.

2.4. Extending the operadic structure on the cells of $\mathcal{A}_{\boldsymbol{g}, \boldsymbol{r}}^{\boldsymbol{s}}$. We can extend the operad structure on $C C_{*}(A, A \backslash \mathcal{A} r c)$ to a partial operad structure on $C C_{*}(A)$. Recall 

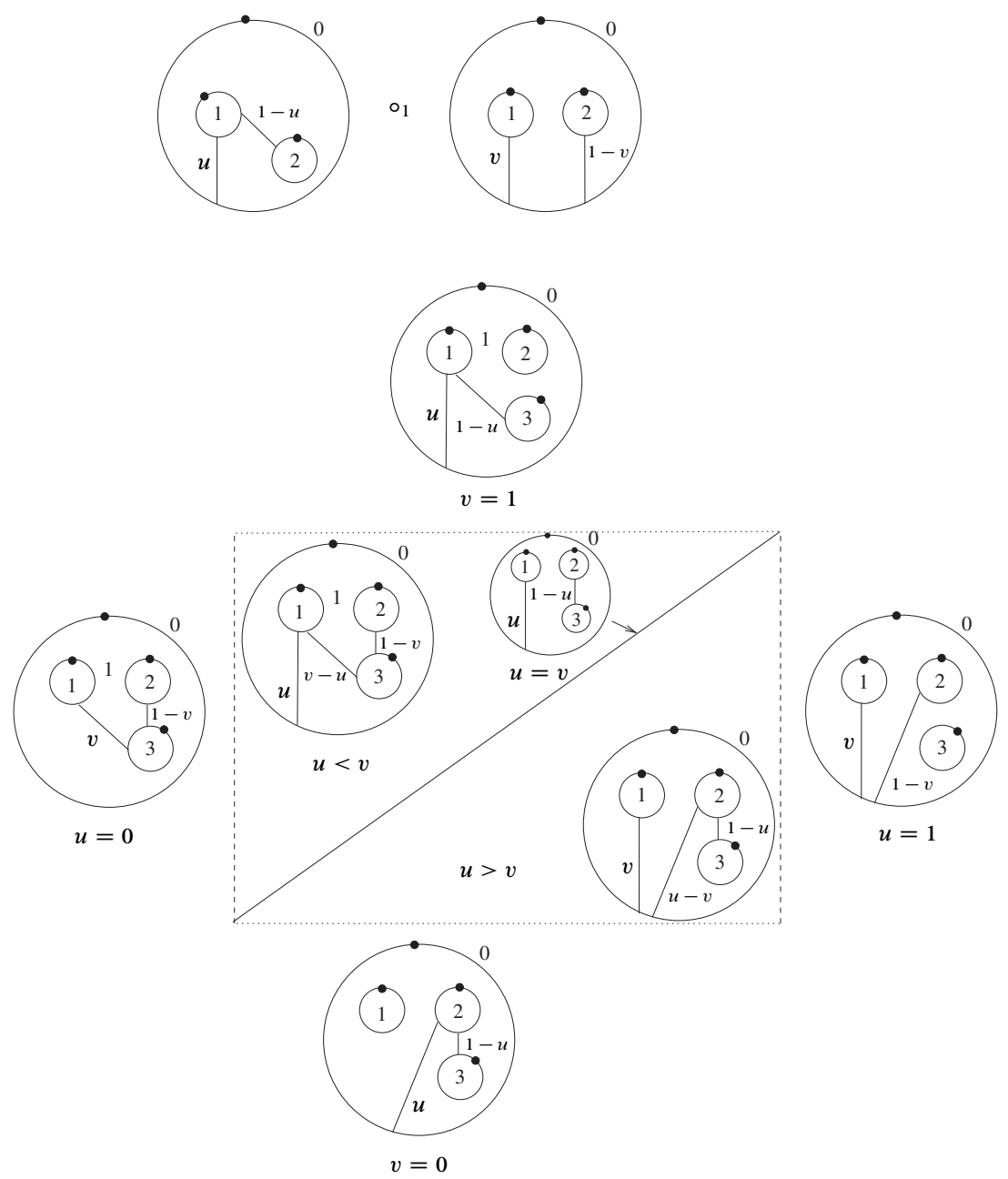

Figure 5. An example of gluing cells. The dashed lines denote the closure of the open cells and the smaller arc graph denotes the codimension one "diagonal" family.

that $\mathcal{A}_{g, r}^{s}$ is a CW complex whose cells are indexed by graphs with possibly isolated vertices. We have to define the gluings for the boundaries with isolated vertices. For two arc graphs $(\Gamma, F,[i]),\left(\Gamma^{\prime}, F^{\prime},\left[i^{\prime}\right]\right)$ we define $\Gamma \circ_{i} \Gamma^{\prime}$ to be given by the induced operation from the topological level if $\Gamma$ has arcs incident to the boundary $i$ and $\Gamma^{\prime}$ has arcs incident to the boundary 0 .

There are more extensions which are natural. If either $\Gamma$ has no arcs incident to the boundary $i$ or $\Gamma^{\prime}$ has no arcs incident to the boundary 0 or both, then we define 
the gluing to be the cell labelled by the graph obtained from $\Gamma$ and $\Gamma^{\prime}$ by deleting all the edges of $\Gamma^{\prime}$ incident to the $i$ th boundary and all the edges incident to $\Gamma$ at the vertex corresponding to the 0th boundary and the vertices at these boundaries. This graph is considered to lie on the surface obtained by gluing the boundary 0 of $F$ to the boundary $i$ of $F^{\prime}$.

2.4.1. An operad structure on $\boldsymbol{C} \boldsymbol{C}_{\boldsymbol{*}}\left(\mathcal{A}_{\boldsymbol{g}, \boldsymbol{r}}^{s}\right)$. We obtain an operad structure on the Abelian groups $C C_{*}\left(\mathcal{A}_{g, r}^{s}\right)$ by taking the above definition in the cases of both boundaries being hit and both boundaries not being hit and setting to zero all other products. This gluing prescription together with the $\mathbb{S}_{n+1}$ action by permutations on the labels indeed gives a cyclic operad structure. We wish to point out that the gluing of empty to empty actually raises the dimension of the cells by one. This effect is due to the $\mathbb{R}_{>0}$ action by scaling, see the discussion of de-projectivized arcs below in §3.2.1.

Proposition 2.17. The gluings on the spaces $C C_{*}\left(\mathcal{A}_{g, r}^{s}\right)$ defined above together with the action of the groups on the labels define a cyclic operad structure. This cyclic operad structure descends to homology inducing a structure of a cyclic operad on $H_{*}(\mathcal{A})(n):=H_{*}\left(\bigsqcup_{s, g} \mathcal{A}_{g, n+1}^{s}\right)$.

Proof. For the first part only the associativity needs to be checked, which is straightforward. For the descent to homology, first notice that for any two classes $\alpha \in$ $C C_{*}(\mathcal{A})(n), \beta \in C C_{*}(\mathcal{A})(m)$,

$$
d\left(\alpha \circ_{i} \beta\right)=d \alpha \circ_{i} \beta \pm \alpha \circ_{i} d \beta \pm d \alpha \circ_{i} d \beta
$$

where the signs are the natural signs induced by the orientations of the cells. The only case which is not straightforward is the case in which the differential deletes the sole edge incident to one of the boundaries involved in the gluing. In this case the composition yields zero, unless there is also only a sole edge incident to the other boundary involved in the gluing. Then and only then does the gluing $d \alpha \circ_{i} d \beta$ not yield zero in the complex, since the dimension goes up by one when gluing two boundary components which do not get hit - as mentioned above - so that the dimension of $d \alpha \circ_{i} d \beta$ is equal to the dimension of $\alpha$ plus the dimension of $\beta$ minus 1 . In this special case, the result of the gluing is the graph obtained from $\alpha \circ_{i} \beta$ by deleting the sole edge intersecting the separating curve which is the image of the glued boundaries.

On the other hand, this is the only summand of $d\left(\alpha \circ_{i} \beta\right)$ that is not a summand of $d \alpha \circ_{i} \beta \pm \alpha \circ_{i} d \beta$. In this case the signs work out as well, since the overall sign stemming from the differentials before gluing and after gluing is the same. Either the number/position of the glued arc is the same as the one on $\alpha$ or as the number/position of the half edge on $\beta$ which does not hit the boundary 0 and in both cases the signs will agree as a straightforward calculation shows. Now for the descent property, choose any two classes $a \in H_{*}(\mathcal{A})(n), b \in H_{*}(\mathcal{A})(m)$. Let $[\alpha]=a$ and $\beta=[b]$ be two 
representatives $d \alpha=d \beta=0$. We wish to set $a \circ_{i} b:=\left[\alpha \circ_{i} \beta\right]$. Due to the relation (2.9), we see that indeed $\left[\alpha^{\prime} \circ_{i} \beta^{\prime}\right]=\left[\alpha \circ_{i} \beta\right]$, for any other representatives $\alpha^{\prime}, \beta^{\prime}$ : $\left[\alpha^{\prime}\right]=[\alpha],\left[\beta^{\prime}\right]=[\beta]$. With this definition, the cyclic structure and associativity are clear.

Examples of the formula (2.9) can be read off from Figures 4 and 5.

Remark 2.18. We wish to point out that in this setting $\operatorname{Gr} \mathcal{C}_{o}^{*}(\mathcal{A} r c)$ presents itself as a mock-dg operad in the sense that the left and right hand side of (2.9) agree even when restricted to $\operatorname{Gr} \ell_{o}^{*}(\mathcal{A} r c)$.

Remark 2.19. We will see below $\S 5.6 .1$ that in a suitable restricted case the mixed gluing can also be used to augment the chain level gluings to a dg structure.

\section{Relations to moduli space and other known operads}

\subsection{Definition of subspaces, suboperads and $\mathscr{D} \mathcal{A} r c$}

3.1.1. Suboperads. We would like to recall and introduce the following notation for subspaces.

\begin{tabular}{|l|l|}
\hline Subspace & Condition \\
\hline $\mathcal{A} r c_{g}^{s}(n) \subset \mathcal{A}_{g, n+1}^{s}$ & $\begin{array}{l}\partial: F_{\Gamma} \rightarrow V_{\Gamma} \text { is surjective. This means that each } \\
\text { boundary gets hit by an arc. }\end{array}$ \\
\hline $\mathcal{A} r c_{\# g}^{s}(n) \subset \mathcal{A} r c_{g}^{s}(n)$ & $\begin{array}{l}\text { The arcs are quasi-filling. This means that com- } \\
\text { plementary regions are polygons or once punc- } \\
\text { tured polygons. }\end{array}$ \\
\hline $\mathcal{T} r e e(n) \subset \mathcal{A} r c(n)$ & $\begin{array}{l}\text { All arcs run only from boundary 0 to some } \\
\text { boundary } i \neq 0 .\end{array}$ \\
\hline $\mathscr{L} \mathcal{T} r e e(n) \subset \mathcal{T} r e e(n)$ & $\begin{array}{l}\text { The linear order of the arcs at the boundary 0 } \\
\text { is anti-compatible with the linear order at each } \\
\text { boundary. I.e. if } \prec_{i} \text { is the linear order at } i \text { then } \\
f \prec_{i} f^{\prime} \text { is equivalent to } l\left(f^{\prime}\right) \prec_{0} l(f) .\end{array}$ \\
\hline $\mathcal{C}$ orol & $\begin{array}{l}\text { Exactly one arc for each boundary } i \neq 0 \text { which } \\
\text { runs to boundary } 0 .\end{array}$ \\
\hline
\end{tabular}


We will use the subscript $c p$ to signify $g=s=0$. Explicitly

$$
\begin{gathered}
\mathcal{A r c}_{c p}(n):=\mathcal{A}_{r} c_{0}^{0}(n), \quad \mathcal{T} r e e_{c p}(n):=\mathcal{T}_{r e e}(n) \cap \mathcal{A} r c_{c p}(n), \\
\mathscr{L} \mathcal{T r e e}_{c p}(n):=\mathscr{L} \mathcal{T}_{r e e}(n) \cap \mathcal{A}_{r} c_{c p}(n), \quad \operatorname{Corol}_{c p}(n)=\operatorname{Corol}(n) \cap \mathcal{A}_{r} c_{c p}(n) .
\end{gathered}
$$

Remark 3.1. One can also define the natural linear order of the flags at the boundary zero to the one which is opposite to the linear order induced by the orientation. This convention is in line with the usual cobordism point of view used in [KLP]. In this case the condition for $\mathscr{L} \mathcal{T}$ ree is the compatibility of the orders.

\subsubsection{De-projectivized arcs}

Definition 3.2. Let $\mathscr{D} \mathcal{A} r c_{g, r}^{s}:=\mathcal{A} r c_{g, r}^{s} \times \mathbb{R}_{>0}$.

The elements of $\mathscr{D A} r c$ are graphs on surfaces with a metric, i.e. a function $w: E_{\Gamma} \rightarrow \mathbb{R}_{>0}$. Furthermore $\mathscr{D} \mathcal{A} r c$ is a cyclic operad equivalent to $\mathcal{A} r c$ [KLP]. The operad structure on $\mathscr{D} \mathcal{A} r c$ is given as follows. Let $\alpha, \alpha^{\prime}$ be elements of $\mathscr{D} \mathcal{A} r c$, if the total weight at the boundary $i$ of $\alpha$ is $\lambda$ and the total weight at the boundary 0 of $\alpha^{\prime}$ is $\mu$, then first scale the metric $w$ of $\alpha$ to $\mu w$ and likewise scale the metric $w^{\prime}$ of $\alpha^{\prime}$ to $\lambda w^{\prime}$ and afterwards glue as above.

Any subspace $\delta$ of the list above defines a suboperad $\mathscr{D} \&:=\delta \times \mathbb{R}_{>0}$ of $\mathscr{D} \mathcal{A} r c$ which is equivalent to $\delta$.

In the above notation one always has isomorphisms of operads $D \mathcal{D} / \mathbb{R}_{>0} \simeq 8$ where $\mathbb{R}_{>0}$ acts by scaling on the right factor $\mathbb{R}_{>0}$ of $\mathscr{D} \mathcal{A} r c$.

Lemma 3.3. Tree, $\mathscr{L}$ Tree and Corol as well as their restrictions to $g=s=0$ are suboperads (not cyclic) of the cyclic operad Arc. The same holds for their versions in $D$ Arc defined above.

Proof. Straightforward, see also [KLP], [K1].

3.2. Relations of subspaces and operads to known operads. It will be convenient for the reader to list the known equivalences of suboperads.

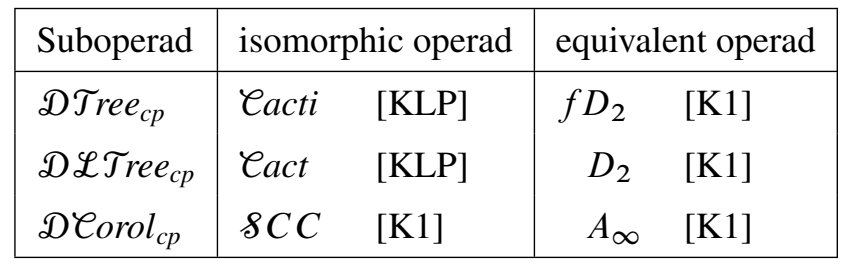

The operads in the third column are the familiar ones, that is $D_{2}$ is the $E_{2}$ operad of little discs, $A_{\infty}$ is the $E_{1}$ operad of little intervals and $f D_{2}$ is the framed little discs operad. The reader unfamiliar with the second column can take the first column as an 
equivalent definition or consult [K1]. Here $8 C C$ is the suboperad of cacti with only one vertex.

Let $M_{g, n+1}^{1^{n+1}}$ be the moduli space of curves of genus $g$ with $n$ punctures and one tangent vector at each puncture. Below we will show that:

\begin{tabular}{l|l} 
& is isomorphic to \\
\hline $\mathcal{D} \mathcal{A} r c_{\#, g}^{0}(n)$ & $M_{g, n+1}^{1^{n+1}}$ \\
$\operatorname{Gr} \mathcal{C}_{o}^{*}\left(\mathcal{A} r c_{\#}^{0}\right)$ & $\mathcal{R} i b$
\end{tabular}

We will first show that the first line is an isomorphism on the level of spaces, and the second on the level of free Abelian groups. As we show below, the collection $\mathscr{D} \mathcal{A} r c_{\#}^{0}(n)$ forms a rational operad. This induces the structure of a rational operad on $M_{g, n+1}^{1^{n+1}}$. We also show that $\operatorname{Gr} \mathcal{C}_{o}^{*}\left(\mathcal{A} r c_{\#}^{0}\right)$ carries an operad structure, which is induced by the operad structure of $\mathcal{A} r c$. This operad structure then carries over to $\mathcal{R} i b$ and hence gives an operad structure on a cell model of $M_{g, n+1}^{1^{n+1}}$, see $\S 2.3$ and $\S 3.1 .1$.

Remark 3.4. The respective quotients by the scaling action or $\mathbb{R}_{>0}$ give rise to equivalent operads.

Remark 3.5. The inclusion of $\mathcal{T} r e e_{c p} \subset \mathcal{A} r c_{\#}$ thus gives an $\mathrm{BV}_{\infty}$ (BV up to homotopy) structure to a cell model of moduli which includes an $A_{\infty}$ structure.

3.2.1. Extended gluing. We can extend the operad structure in $D \mathcal{A}$ r $r c$ on the spaces $D_{\mathcal{A}_{g, r}^{s}}^{s}:=\mathcal{A}_{g, r}^{s} \times R_{>0}$ as follows. If both the boundaries that are to be glued are hit, then glue as above. If none of the two boundaries to be glued is hit, define the composition to be given by the image of the two arc graphs on the glued surface minus the two vertices corresponding to the two boundaries that are glued together. Lastly if one of the boundaries is empty, then we delete the vertices corresponding to the two boundaries that are to be glued along with all the edges incident to these vertices. The latter operation is not in general associative, but it can be shown that we obtain an operad up to homotopy or a quasi-operad (see [K1] of Definition 5.22 below). These operations induce the extended chain operations on the space $C C_{*}(A)$ which are discussed in \$2.4. The operations of gluing boundaries which are hit to boundaries which are not hit still only yield a partial operad structure, however, since we have to take care that there is at least one arc left. To obtain an unrestricted operad structure one has to allow graphs without any edges. We can include the empty arc family as a point in $\mathscr{D} \mathcal{A}_{g, r}^{s}$ as the image of the origin in $\mathbb{R}_{\geq 0}^{n}$, that is the family whose weights are all zero. We define the space $\mathscr{D} \mathcal{A}_{g, r}^{s}$ to be $\mathscr{D}_{\mathcal{A}}^{s, r}:=\mathcal{A}_{g, r}^{s} \cup\{\oslash\}$ where the topology is defined by considering $\oslash$ as the limit in which all weights of the edges go to zero. This space is obviously contractible to $\oslash$ by scaling all the weights on the edges of a given graph homogeneously down to zero. Summing up, we obtain: 
Lemma 3.6. There are contractible spaces $\mathscr{D}_{\mathcal{A}}$ which form a quasi-operad whose structure maps induce the operad structure of $\mathcal{A}$ and $\mathcal{A}$ rc.

3.3. The duality between quasi-filling arcs and ribbons graphs. In [KLP] we defined a map called $\mathscr{L}$ oop which is the suitable notion of a dual graph for a graph on a surface. This map uses an interpretation of the graph as a partially measured foliation. If one restricts to the subspace $\mathcal{A} r r_{\#}$ though, this map has a simpler purely combinatorial description. This description will be enough for our purposes here, but we would like to emphasize that this description is only valid on the subspace $\mathcal{A} r c_{\#}$ and cannot be generalized to the whole of $\mathcal{A} r c$ unlike the map $\mathscr{L}$ oop. Figure 6 contains an example of an arc graph and its dual.

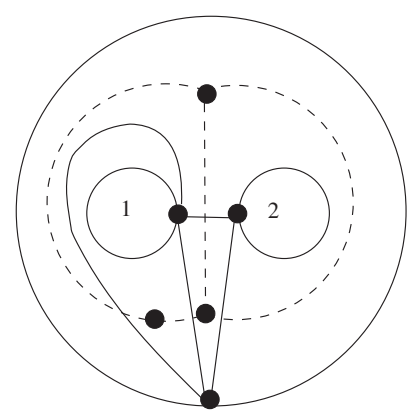

Figure 6. An example of an arc graph (solid) and its dual (dashed).

3.3.1. The dual graph. Informally the dual graph of an element in $\mathcal{A} r c_{\#}$ is given as follows. The vertices are the complementary regions. Two vertices are joined by an edge if the complementary regions border the same arc. Due to the orientation of the surface this graph is actually a ribbon graph via the induced cyclic order. Moreover the marked points on the boundary make this graph into a marked ribbon graph. A more precise formal definition is given in the next few paragraphs.

3.3.2. Polygons and $\mathcal{A} r \boldsymbol{c}_{\#}$. By definition, in $\mathcal{A} r \boldsymbol{r}_{\#}$ the complementary regions are $k$-gons or once punctured $k$-gons. Let $\operatorname{Poly}(F, \Gamma, \overline{[i]})$ be the set of these polygons and let $\operatorname{Sides}(F, \Gamma, \overline{i i]})$ be the disjoint union of sets of sides of the polygons. We define $\partial_{\text {poly }}: \operatorname{Sides}(F, \Gamma, \overline{[i]}) \rightarrow \operatorname{Poly}(F, \Gamma, \overline{[i]})$ to be the map which associates to a side $s$ of a polygon $p$ the polygon $p$. The sides are either given by arcs or the boundaries. We define the map Lab: $\operatorname{Sides}(F, \Gamma, \overline{[i]}) \rightarrow E_{\Gamma} \cup V_{\Gamma}$ that associates the appropriate label. Notice that $\left|\mathrm{Lab}^{-1}(e)\right|=2$ for $e \in E_{\Gamma}$ and that $\left|\mathrm{Lab}^{-1}(v)\right|=1$ for $v \in V_{\Gamma}$. Thus there is a fixed point free involution $l_{\text {side }}$ on the set $\mathrm{Lab}^{-1}\left(E_{\Gamma}\right)$ of sides of the polygons marked by arcs which maps one side to the unique second side carrying the same label. This in turn defines an involution $l$ of pairs $(p, s)$ of a polygon together 
with a side in $\mathrm{Lab}^{-1}\left(E_{\Gamma}\right)$ by mapping $s$ to $l_{\text {side }}(s)$ and taking the polygon $p$ to the polygon $p^{\prime}:=\partial_{\text {poly }}(l(s))$ of which $l_{\text {side }}(s)$ is a side. Although $p$ and $p^{\prime}$ might coincide the sides will differ making the involution $\imath$ fixed point free.

3.3.3. The dual graph of an element of AArc. For an element $\alpha=(F, \Gamma, \overline{[i]}, w) \in$ $\mathcal{A r c}_{\#}(n)$ we define the dual graph to be the marked ribbon graph with a projective metric $\hat{\Gamma}(\alpha):=(\widehat{\Gamma}, \widehat{w}, \mathrm{mk})$ and $\mathbb{Z} / 2 \mathbb{Z}$ marking on the vertices pct which is defined as follows. The vertices of $\widehat{\Gamma}$ are the complementary regions of the arc graph (i.e. the polygons) and the map pct associates to a vertex the number 1 if the complementary region is punctured and 0 if it is not. The flags of the graph are the pairs $(p, s)$ of a polygon (vertex) together with a side of this polygon marked by an arc $(s \in$ $\left.\mathrm{Lab}^{-1}\left(E_{\Gamma}\right)\right)$. The map $\partial$ is defined by $\partial((p, s))=p$ and the involution $l((p, s)):=$ $\left(\partial_{\text {poly }}\left(l_{\text {side }}(s)\right), l_{\text {side }}(s)\right)$.

Each polygonal complementary region is oriented by the orientation induced by the surface, so that the sides of each polygon and thus the flags of $\hat{\Gamma}$ at a given vertex $p$ have a natural induced cyclic order making $\widehat{\Gamma}$ into a ribbon graph.

Notice that there is a one-one correspondence between edges of the dual graph and edges of $\Gamma$. This is given by associating to each edge $\{(p, s), \imath(p, s)\}$ the edge corresponding to the $\operatorname{arc} \operatorname{Lab}(s)$.

We define a projective metric $\hat{w}$ on this graph by associating to each edge $\{(p, s), l(p, s)\}$ the weight of the arc labelling the side $s: \hat{w}(\{(p, s), l(p, s)\}):=$ $w(\operatorname{Lab}(s))$, where $w$ is the projective metric on the arc graph.

To define the marking of the ribbon graph, we first notice that the cycles of $\hat{\Gamma}$ correspond to the boundary components of the surface $F$. Let $c_{k}$ be the cycle of the boundary component labelled by $k$. The $k$-th boundary component then lies in a unique polygon $p=\partial_{\text {poly }}\left(\mathrm{Lab}^{-1}(k)\right)$. Let $\prec_{p}$ be the cyclic order on the set of sides of $p, \partial^{-1}(p)$. Let $s_{k}$ be the side corresponding to the boundary and let $\curvearrowright\left(s_{k}\right)$ the element following $s_{k}$ in $\prec_{p}$. We define $\operatorname{mk}\left(c_{k}\right):=\left(p, \curvearrowright\left(s_{k}\right)\right)$.

Remark 3.7. The space $\mathcal{A} r c_{\#}^{0}$ corresponds to graphs with pct $\equiv 0$ and we will omit mention of the function pct for these graphs.

The above map will suffice for the purposes of this paper. For the general theory and the reader acquainted with the constructions of [KLP], the following will be helpful.

Remark 3.8. For elements in $\mathcal{A} r c_{\#}^{0}$ the dual graph realizes the map $\mathscr{L}$ oop of [KLP], i.e. for $(F, \Gamma, \overline{[i]}, w) \in \mathcal{A} r c_{\# g}^{0}(n), \mathscr{L} \operatorname{oop}(F, \Gamma, \overline{[i]}, w)=(\hat{\Gamma}$, ord $, \hat{w}, \mathrm{mk})$.

3.3.4. From marked weighted ribbon graphs to arc families. Given a marked weighted ribbon graph $\Gamma$, we fix $F=\Sigma(\Gamma)$. The boundary components of $F$ correspond to the cycles of $\Gamma$ and thus the former are labelled if the latter are. If $c_{i}$ 
is a cycle of $\Gamma$ we denote the corresponding boundary by $\partial_{i} F$. Let $\widehat{G}$ be the dual graph on the surface of $\Gamma$. This graph can be constructed as follows. Let $\Gamma \subset F$ be embedded as the spine. For each edge $e=\{f, l(f)\}$ with $f \in c_{i}$ and $l(f) \in c_{j}$. Fix the mid-point of each edge $\operatorname{mid}(e)$. Now let $\hat{e}$ be the $\operatorname{arc} \operatorname{mid}(e) \times I$ on $F$. This edge is broken into two flags $\hat{f},(\hat{f})$ by the midpoint of the interval. Here the flag which is named $\hat{f}$ is the flag of $\hat{e}$ which runs from the midpoint $\operatorname{mid}(e)$ to the boundary $\partial_{i} F$. This defines a map $\hat{:} F_{\Gamma} \rightarrow F_{\hat{\Gamma}}$. It then follows that $l \widehat{(f)}=\imath(\hat{f})$. From $\hat{\Gamma}$ it is easy to obtain an element of $\mathcal{A} r c$ in the picture where the arcs do not run to the marked point on the boundary. Just mark a point on each boundary $\partial_{i} F$ such that the linear order of incident edges to $\partial_{i} F$ is that of the cycle $c_{i}$. For instance any point which is slightly before the point of intersection of $\widehat{\mathrm{mk}\left(c_{i}\right)}$. To obtain a graph on $F$ in the sense of $\S 2$, let $p_{i} \in \partial_{i} F$ be the endpoint of $\widehat{\operatorname{mk}\left(c_{i}\right)}$ and choose a homotopy of the flags $\hat{f}$ which fixes the point $\operatorname{mid}(e)$ and slides all the endpoints on the various $\partial_{i} F$ to fixed point $p_{i} \in \partial_{i} F$ in the direction opposite to the natural orientation of $\partial_{i} F$. Obviously the homotopy can be chosen, such that all the arcs will be embedded. The edges which are now called arcs incident to $p_{i}$ will then have a linear order starting with the edge to which $\widehat{\mathrm{mk}\left(c_{i}\right)}$ belongs.

Proposition 3.9. The dual map identifies the space $\mathcal{D} \mathcal{A} r c_{\#}^{0}$ with the space of metric marked ribbon graphs $\mathcal{M} R i b$. Moreover this correspondence is on the level of $\mathbb{R}_{>0}$ spaces where the action on $\mathcal{M} \mathcal{R} i b$ is by rescaling the metric, i.e., Arr $c_{\#}$ and $\mathbb{P} \mathcal{R} i b$ are identified by the dual graph construction.

Proof. As seen above we have maps $\phi: \mathscr{D} \mathcal{A} r c_{\#}^{0} \rightarrow \mathcal{M} \mathcal{R} i b$ and $\psi: \mathcal{M} \mathcal{R} i b \rightarrow$ $\mathscr{D} \mathcal{A} r c_{\#}^{0}$. It is straightforward to check that $\phi$ and $\psi$ are inverses, since the surface of an element in $\mathscr{D} \mathcal{A} r c_{\#}^{0}$ can uniquely be reconstructed from the underlying graph.

Remark 3.10. If we regard pairs ( $\Gamma$, pct) then we obtain a map to $\mathscr{D} \mathcal{A} r c_{\#}$ where in the last step we put a puncture at each vertex $v$ which is has $\operatorname{pct}(v)=1$. There is no operad structure however, since we cannot guarantee that the number of punctures does not increase.

Of course, we could keep track of this and regard the subspace $\mathcal{A} r{ }_{\mathrm{C} \# \#}$ of elements of $\mathcal{A} r c$ whose complementary regions are polygons, or multiply punctured polygons. Then the function pct would take values in $\mathbb{N}$ and we would obtain analogous statements to the Proposition 3.9 and Corollary 4.4.

3.4. Ribbon graphs and moduli space. The spaces $\mathcal{A} r \mathrm{C}_{g}^{s}(n)$ are related to moduli spaces of Riemann surfaces with extra structures. This for instance follows using Strebel differentials [St] to relating the $\mathcal{A} r c$ to the moduli space of curves with marked points and tangent vectors at these points. 
Theorem 3.11. The space $\mathscr{D} \mathcal{A} r c_{g}^{0}(n)$ and the moduli space $M_{g, n+1}^{1^{n+1}}$ of $n+1$ punctured Riemann surfaces of genus $g$ with one tangent vector fixed at puncture each are isomorphic in the coarse moduli or orbifold sense. Moreover they are isomorphic as $\mathbb{R}_{>0}$ spaces where the action on $M_{g, n+1}^{1^{n+1}}$ is given by simultaneous rescalings of all tangent vectors, i.e., $\mathcal{A} r c_{\#}^{0} \simeq M_{g, n+1}^{1^{n+1}} / \mathbb{R}_{>0}:=\mathbb{P} M_{g, n+1}^{1^{n+1}}$.

Proof. As shown each $\alpha \in \mathscr{D} \mathcal{A} r c_{g}^{0}(n)$ uniquely corresponds to a marked ribbon graph with a metric, which is embedded as the spine of the given surface. Now as usual by gluing in punctured discs to the boundaries using Strebel differentials, we obtain a punctured surface. Now, furthermore, we retain the length of the boundary cycle and a tangent direction. This direction is given by the direction vertical trajectory which hits the marked point on the boundary. This data gives rise to a surface with a marked point and a tangent vector at each boundary, by decomposing $v \in T_{p_{i}} F$ as $|v| e_{v}$ with $e_{v} \in T_{p_{i}}^{1} F$. It is clear that this assignment is a bijection in view of the known characterization of combinatorial moduli space [St], [Ko1], [Ko2], [HM], [H], [P], [P2], [CV]. Moreover this correspondence is compatible with the orbifold topology or the coarse moduli structure. The statement about the $\mathbb{R}_{>0}$ action is obvious from the description.

Corollary 3.12. There is a decomposition of $M_{g, n+1}^{1^{n+1}}=\bigsqcup_{\Gamma \in \mathcal{R} i b} \dot{C}(\Gamma)$ with $\dot{C}(\Gamma)$ open cells indexed by marked ribbon graphs.

Remark 3.13. Alternatively, one could use Penner's formalism [P2] in the hyperbolic setup to get a proper homotopy equivalence between $\mathcal{A} r c_{\#}(F)$ and $M(F) / \mathbb{R}_{>0}$ for any bordered surface $F \neq F_{0,2}^{0}$. Here $M(F)=\left[\operatorname{Hyp}(F) \amalg\left(\prod_{1}^{r} \partial_{i}\right)\right] / \sim$ where $\sim$ is the equivalence relation generated by the push-forward of the metric under orientationpreserving diffeomorphism and $\operatorname{Hyp}(F)$ is the space of all hyperbolic metrics with geodesic boundary on the surface $F$ whose boundary components are the $\partial_{i}$.

3.4.1. The graph complex and the mapping class group. By using the ribbon graph interpretation for $\mathcal{C}_{o}^{*}\left(\mathcal{A} r r_{\#}\right)(F)$ and its differential we obtain the graph complex of marked ribbon graphs. Furthermore its homology computes the cohomology of the mapping class group of $F$ by the usual arguments [Ko2], [P2], [P], [CV].

Notation 3.14. We let $\mathcal{C}_{o}^{*}\left(\mathcal{A} r C_{\#}^{0}\right)$ be the subgroup of $\mathcal{C}_{o}^{*}(\mathcal{A} r c)$ generated by the cells corresponding to quasi-filling arc families with no punctures and write $\mathrm{Gr}_{\boldsymbol{o}}^{*}\left(\mathcal{A} r c_{\#}^{0}\right)$ for the image of this subgroup on $\operatorname{Gr} \mathcal{C}_{o}^{*}(\mathcal{A} r c)$.

Definition 3.15. The graph complex of marked ribbon graphs is the Hopf algebra whose primitive elements are connected marked ribbon graphs and whose product is the disjoint union. Its differential is given by the sum of contracting edges $d \Gamma=$ 
$\sum_{e \in E^{\prime}(\Gamma)} \pm \Gamma / e$, where $E^{\prime}(\Gamma)$ is the subset of edges $e$ such that the topological type of $\Gamma$ coincides with that of $\Gamma / e$ and the sign is the usual sign.

Proposition 3.16. The following two complexes are isomorphic:

i) $\left(\operatorname{Gr} \mathcal{C}_{o}^{*}\left(\mathcal{A} r c_{\#}^{0}\right)(F), d\right)$,

ii) graph complex of marked ribbon graphs;

and they both compute $H^{*}(P M C(F))$, the cohomology of the pure mapping class group and the spaces $H^{*}\left(M_{g, n+1}^{n+1}\right)$.

Proof. The differential of the graph complex is the signed sum over those contractions of edges, which leave the topological type of the graph (genus and number of boundaries) intact. This is exactly dual the differential of $\mathcal{C}_{o}^{*}\left(\mathcal{A} r c_{\#}^{0}\right)$ which deletes the respective arcs. The fact that they both compute $H^{*}(P M C(F))$ is essentially the Kontsevich-Penner theorem adapted to the case with boundary. The proof is a standard application of the techniques of [Ko2], [P2], [P], [CV].

\section{The cell operad of moduli space}

Notice that the gluing operation of $\mathcal{A} r c$ applied to two elements of $\mathcal{A} r c_{\#}^{0}$ need not land in $\mathcal{A} r c_{\#}^{0}$. Generically, however, that is inside the top-dimensional cells, two elements in $\mathcal{A} r c_{\#}^{0}$ do glue to form an element in $\mathcal{A} r c_{\#}^{0}$. In order to deal with this situation, we introduce the following notion.

Definition 4.1. A rational topological operad is a collection of topological $\mathbb{\$}_{n}$ modules together with operadic structure maps which only need to be defined and continuous on a dense subset. These structure maps are required to satisfy the operad axioms where they are defined.

We will call a rational operad almost topological if the structure maps of the rational operad can be extended to the whole space in a possibly non-continuous fashion, such that forgetting the topology, the induced structure is an operad of sets.

Theorem 4.2. A $A r c_{\#}^{0}$ is a rational topological operad and $\operatorname{Gr} \mathcal{C}_{o}^{*}\left(\mathcal{A} r c_{\#}^{0}\right) \subset$ $\mathrm{Gr} C_{o}^{*}($ Arc $)$ is a cyclic suboperad which is a dg operad.

Proof. We will show that the top-dimensional cells in the composition of two cells from $\mathcal{C}_{o}^{*}\left(\mathcal{A} r c_{\#}^{0}\right)$ are also cells of $\mathcal{C}_{o}^{*}\left(\mathcal{A} r c_{\#}^{0}\right)$. This can be done with the help of the Euler characteristic. First notice that the top-dimensionality of the cells implies that there are no closed loops and that we are in the generic situation for the gluing as described in §2.2. Notice that by Lemma 2.8 we only have to show that the Euler characteristic of the arc family is the Euler characteristic of the glued surface. Now gluing the arc 
families supported on the surfaces $F_{1}$ and $F_{2}$, say, we obtain a graph $\Gamma$ on the glued surfaces which we call $F$. Let $S$ be the separating curve which constitutes the glued boundaries of the two glued surfaces $F_{1}$ and $F_{2}$. Now the Euler characteristic satisfies $\chi(F)=\chi\left(F_{1}\right)+\chi\left(F_{2}\right)$. Let $\Gamma_{1}$ on $F_{1}$ and $\Gamma_{2}$ on $F_{2}$ be the graphs before gluing. Fix a partition or $(k, l)$-shuffle (cf. §2.2.6) indexing a top-dimensional cell and let $\Gamma_{1}^{\prime}$ and $\Gamma_{2}^{\prime}$ be the graphs obtained from $\Gamma_{2}$ and $\Gamma_{1}$ by inserting parallel $k+l$ edges and breaking up the vertices 0 and $i$ according to this partition in the gluing procedure of $\$ 2.2$. See Figure 3 for an example. Let $\Gamma^{\prime}$ be the graph consisting of $\Gamma_{1}^{\prime}, \Gamma_{2}^{\prime}$ and the curve $S$. It is clear that $\Gamma \subset \Gamma^{\prime}$.

The graph $\Gamma$ is obtained from $\Gamma^{\prime}$ by erasing the edges belonging to $S$. We claim that

$$
\chi\left(\Gamma^{\prime}\right)=\chi\left(\Gamma_{1}\right)+\chi\left(\Gamma_{2}\right)
$$

and that

$$
\chi\left(\Gamma^{\prime}\right)=\chi(\Gamma) .
$$

Assuming these equalities we obtain $\chi(F)=\chi\left(F_{1}\right)+\chi\left(F_{2}\right)=\chi\left(\Gamma_{1}\right)+\chi\left(\Gamma_{2}\right)=$ $\chi\left(\Gamma^{\prime}\right)=\chi(\Gamma)$, whereby $\chi(F)=\chi(\Gamma)$ and hence by Lemma $2.8 \Gamma$ is quasi-filling. To validate (4.1) we calculate: $V\left(\Gamma^{\prime}\right)=V\left(\Gamma_{1}\right)+V\left(\Gamma_{2}\right)+(k+l-1), E\left(\Gamma^{\prime}\right)=$ $E\left(\Gamma_{1}\right)+E\left(\Gamma_{2}\right)+2(k+l-1)$ and $\operatorname{Cyc}(\Gamma)=\operatorname{Cyc}\left(\Gamma_{1}\right)+\operatorname{Cyc}\left(\Gamma_{2}\right)+k+l-1$ so that $V\left(\Gamma^{\prime}\right)-E\left(\Gamma^{\prime}\right)+\operatorname{Cyc}\left(\Gamma^{\prime}\right)=V\left(\Gamma_{1}\right)-E\left(\Gamma_{1}\right)+\operatorname{Cyc}\left(\Gamma_{1}\right)+V\left(\Gamma_{2}\right)-E\left(\Gamma_{2}\right)+\operatorname{Cyc}\left(\Gamma_{2}\right)$. To check (4.2), we first need to make some observations. First, the curve $S$ breaks up into the pieces corresponding to the subintervals of $\mathcal{P}_{\circ}$ of $\$ 2.2 .6$ in their order - which we will call intervals - and one piece which is between the first and the last arc - which we will call the outside arc of $S$. Secondly, the complementary regions of $\Gamma^{\prime}$ correspond to those of $\Gamma_{1}, \Gamma_{2}$, triangles corresponding to non-recursive subintervals and quadrangles corresponding to recursive subintervals. Notice that at least on one side of an interval is a piece which has parallel flags, viz. a quadrangle or a triangle, since we are in the maximal dimension and we have a full partition. Moreover if a subinterval is the boundary between a polygon and a quadrangle, then on the opposite side of this quadrangle will be another quadrangle or triangle, since the flags will remain parallel. Now because of these observation, we see that removing the intervals does not change the Euler characteristic. Moreover, we see that no two of the original polygons of $\Gamma_{1}$ and $\Gamma_{2}$ have been joined when removing the intervals. So finally by removing the outside arc of $C$ one effectively glues two distinct polygons together and hence again does not change the Euler characteristic.

For the dg part, we notice that in view of the considerations above if an $\operatorname{arc}$ of $\Gamma$ is not one of the glued ones then the condition that it is removable - viz. the condition that the remaining graph is quasi-filling - is the same before and after the gluing. Moreover the same holds true for the arcs which are glued. If a weight of one of these goes to zero, then this corresponds to a face of the simplex defined by $\Gamma$, cf. §2.2.6. If this face is allowed in $\Gamma$, that is $\Gamma$ after deleting this edge is still quasi-filling, the 
respective limit is allowed by the above in $\Gamma_{1}$ and $\Gamma_{2}$. The same holds true for all the iterations of passing to faces.

Notice that in the quasi-filling case the limit which appeared in $\$ 2.16$ as a counterexample to the compatibility of the operad structure with the differential in the general case is not valid anymore since the relevant limit is neither allowed for $\Gamma_{1}$ nor for $\Gamma_{2}$ inside $\mathcal{A} r c_{\#}^{0}$.

Remark 4.3. The equation (4.1) above also holds in the case that both the families are twisted at the boundaries which are glued.

Corollary 4.4. D $\mathcal{A} r c_{\#}$ and $M_{g, n+1}^{1^{n+1}}$ are a rational operads. Moreover there is an operad structure on the free Abelian group generated by ribbon graphs $\mathcal{R} i b$ which is induced via the identification $\mathcal{R} i b \simeq \mathcal{C}_{o}^{*}\left(\mathcal{A} r c_{\#}^{0}\right) \simeq \mathrm{Gr} \mathcal{C}_{o}^{*}\left(\mathcal{A} r c_{\#}^{0}\right)$. That is there is a dg operad structure on the graph complex computing the cohomology of $M_{g, n+1}^{1^{n+1}}$.

\section{Di-operads and PROPs based on the Arc operad}

Notation 5.1. In all the following the subscript \# will mean that the condition that arc families under consideration are quasi-filling.

Convention. In this paragraph, we will also restrict to the case $s=0$ when we restrict to the quasi-filling case.

5.1. The di-operad $\mathcal{A} \boldsymbol{r} \boldsymbol{c}^{i / \boldsymbol{o}}$. We will consider additional markings for elements of the $A r c$ operad. The first is a partition of $\bar{n}=$ In $\amalg$ Out. Such a partition is equivalent to a map $i / o: \bar{n} \rightarrow \mathbb{Z} / 2 \mathbb{Z}$ where $\operatorname{In}=i / o^{-1}(1)$ and Out $=i / o^{-1}(0)$. Let Part $(n)$ be the space of maps from $\{0, \ldots, n\}$ to $\{0,1\}$ and let $\operatorname{Part}(k, l) \subset \operatorname{Part}(k+l-1)$ be the subset of functions with $|\operatorname{In}|=k, \mid$ Out $\mid=l$.

Let $\mathbb{Z} / 2 \mathbb{Z}[1]=\{\mathbb{Z} / 2 \mathbb{Z}[1](n)\}$ be cyclic operad of spaces built on $\mathbb{Z} / 2 \mathbb{Z}$, that is, the operad of spaces as defined in $[\mathrm{K} 1]$ shifted by 1 . Then $\mathbb{Z} / 2 \mathbb{Z}[1](n)=$ $\mathbb{Z} / 2 \mathbb{Z}(n+1)=(\mathbb{Z} / 2 \mathbb{Z})^{\times n+1}$ where the indexing set for the Cartesian product is taken to be $\bar{n}=\{0, \ldots, n\}$ and the action of $\mathbb{\Phi}_{n+1}$ is by permutations.

Set $\mathcal{A} r c^{i / o}(n, m):=\operatorname{A} r c(n+m-1) \times \operatorname{Part}(n, m)$. Identifying $\operatorname{Part}(n, m)$ with $\mathbb{Z} / 2 \mathbb{Z}^{n+m-1}$ and restricting the gluing in the cyclic A $r c$ operad to only gluing "ins" to "outs" and retaining the in/out designation on the non-glued boundaries one immediately obtains:

Proposition 5.2. Let $\mathcal{A} r c^{i / o}$ be the collection of $\mathbb{\$}_{n} \times \mathbb{\$}_{m}$ modules $\mathcal{A} r c^{i / o}(n, m)$ where the action of the symmetric groups is the action of permuting the labels of the In and Out boundaries, then is Arc ${ }^{i / o}$ a di-operad. It is isomorphic to a partial 
suboperad of the direct product of operads $\mathcal{A} r c \times \mathbb{Z} / 2 \mathbb{Z}[1]$. Furthermore $\mathcal{A} r c_{\#}^{i / o, 0}$ is a sub-di-operad on the cellular level, i.e. the respective subgroups in $\mathrm{Gr}^{\mathcal{C}_{o}^{*}}\left(\mathcal{A} r c_{\#}^{0}\right)(n) \times$ $(\mathbb{Z} / 2 \mathbb{Z})^{n+1}$ form a di-operad.

Definition 5.3. We define the following subspaces (recall that the subscript \# also implies $s=0$ in this section).

\begin{tabular}{|l|l|}
\hline Subspace & Condition \\
\hline $\mathcal{A} r c^{i \leftrightarrow i}(n, m) \subset \mathcal{A} r c^{i / o}(n, m)$ & $\begin{array}{l}\text { Arcs from input to output boundaries } \\
\text { or from output to output boundaries } \\
\text { only. Viz. no arcs from input to input. }\end{array}$ \\
\hline $\mathcal{A} r c^{i \leftrightarrow o}(n, m) \subset \mathcal{A} r c^{i \leftrightarrow i}(n, m)$ & $\begin{array}{l}\text { Arcs only from input to output bound- } \\
\text { aries. }\end{array}$ \\
\hline $\mathcal{A} r c^{\mathrm{Sul}}(n, m) \subset \mathcal{A} r c_{\#}^{i \leftrightarrow i}(n, m)$ & $\begin{array}{l}\text { After removing all arcs which run } \\
\text { from out to out the arc family is still } \\
\text { quasi-filling. }\end{array}$ \\
\hline $\mathcal{A} r c^{\mathrm{StSul}}(n, m) \subset \mathcal{A} r c^{\mathrm{StSul}}(n, m)$ & $\begin{array}{l}\text { Arc families such that no two neigh- } \\
\text { boring arcs on an "out" boundary both } \\
\text { run to "in" boundaries. }\end{array}$ \\
\hline
\end{tabular}

\subsection{Relation to graphs and Sullivan chord diagrams}

Proposition 5.4. The dual graphs of $\mathcal{A} r c_{\#}^{i \leftrightarrow o}(n, m)$ lie in $\mathcal{R} i b^{i \leftrightarrow o}(n, m)$, the dual graphs of $\mathcal{A r c} c^{\mathrm{StSul}}(n, m)$ lie in Chord ${ }^{\mathrm{StSul}}$, and the dual graphs of $\mathcal{A} r c^{\mathrm{Sul}}(n, m)$ lie in Chord ${ }^{\text {Sul }}(n, m)$. Moreover the mentioned subspaces of A rrc $^{i \leftrightarrow o}$ are naturally identified with the relevant subspaces of $\mathbb{P} \mathcal{R} i b^{i / o}$ of graphs with a projective metric. Therefore the cells of $\mathcal{C}_{o}^{*}\left(\mathcal{A} r \mathrm{c}^{i / o}\right) \simeq \mathrm{Gr} \mathcal{C}_{o}^{*}\left(\mathcal{A} r \mathrm{c}^{i / o}\right)$ belonging to these subspaces are exactly indexed by the graphs of the indicated type.

Proof. The claim about the graphs becomes clear by unravelling the dual graph construction. A dual edge to an arc which runs from in to out is an edge which is part of an In and an Out cycle. Likewise an arc from out to out yields an edge which belongs to two (not necessarily distinct) Out cycles. The condition that the out-out edges form trees is equivalent to the fact that contracting them does not change the genus and the number of cycles. In other words the contracted graph and the graph define the same topological surface. Contraction is dual to deletion, hence the condition to be quasi-filling is dual to the condition of contractibility. The condition on Sullivan chord diagrams in the strict sense is that the In cycles are disjointly embedded. This means that no two In cycles share a vertex, i.e., there is at least one out-out edge 
between them. The dual to this condition is the one that is stated. Now the rest of the statements directly follow.

Remark 5.5. Just like $\mathcal{A} r r_{\#}$, the subspaces $\mathcal{A} r c^{\mathrm{StSul}}$ and $\mathcal{A} r r^{\mathrm{Sul}}(n, m)$ are not stable under composition, since the condition of non-topology changing contractibility of the out-out arcs is not stable under the composition.

Remark 5.6. It is clear that $\mathcal{A} r c^{\mathrm{Sul}}(n, m)$ retracts to its subspace $\mathcal{A} r c_{\#}^{i \leftrightarrow o}$ by the homotopy that homogeneously scales all weights on the arcs from "outs" to "outs" to zero.

Just as the respective graphs, see Remark 1.6, $\mathcal{A} r c_{\#}^{i \leftrightarrow o}(n, m)$ and $\mathcal{A} r c^{\mathrm{StSul}}(n, m)$ are weakly homotopy equivalent.

Proposition 5.7. The collections of $\mathbb{S}_{n} \times \mathbb{S}_{m}$ modules, $\mathcal{A} r c^{i \leftrightarrow i}(n, m)$, A $A r c^{i \leftrightarrow o}$ form di-operads.

The subspaces $\mathcal{A} r c_{\#}^{i \leftrightarrow i}$ and $\mathcal{A} r c_{\#}^{i \leftrightarrow o}$ are rational sub-di-operads which induce $d g$ di-operads on the graded open-cell operad level. Hence there is an induced dg di-operad structure on the respective graphs.

Proof. On the topological level, we only have to show that the gluing preserves the subspaces. In the operadic composition, the arcs/bands are matched or split and then matched. In both cases an arc which runs from in to out will be continued with an arc from in to out and thus will run from in to out. In the case of $\mathcal{A r c}^{i \leftrightarrow i}$ an arc which runs from out to out might be matched with an arc running from in to out. The resulting arc will again run from out to out. Therefore the di-operad structure follows from the operad structure of $\mathcal{A} r c$.

Lastly, the claims about the open cell level all follow from Proposition 4.2, the remarks above and the fact that $\mathrm{Gr} \mathcal{C}_{o}^{*}\left(\mathcal{A} r c_{\#}^{0}\right)$ is a dg operad and hence the respective boundary limits before and after gluing coincide.

5.2.1. Standard In/Out markings. There are no natural partitions into in and output boundaries for general elements of $\mathcal{A} r c$, except all inputs or all outputs; it is after all a cyclic operad. If one has a family, however, which has a partition of the boundary $S_{1} \amalg S_{2}=\{0, \ldots, n\}$ such that the arcs of this element only run between $S_{1}$ and $S_{2}$ then one has a $\mathbb{Z} / 2 \mathbb{Z}$ choice of calling $S_{1}$ either In or Out. In this case, we will fix that the set containing 0 will be called Out. This establishes an identification of $\mathcal{A} r c^{i \leftrightarrow o}$ with a partial suboperad of $\mathcal{A} r c$. Also there is a partition of the boundary $S_{1} \amalg S_{2}=\{0, \ldots, n\}$ such that the arcs of this element only run between $S_{1}$ and $S_{2}$ or between $S_{2}$ and $S_{2}$ and there is at least one such arc; we set $S_{2}=$ Out and hence identify $\mathcal{A} r c^{i \ll i}$ with a partial suboperad of $\mathcal{A} r c$.

The subspace $\mathcal{T}$ ree is also of the form described above. Hence the boundaries have a standard $\mathbb{Z} / 2 \mathbb{Z}$ marking with $i / o(0)=0$ and $i / o(j)=1$ for $j \neq 0$. This identifies $\mathcal{T}$ ree $\subset \mathcal{A} r c^{i \leftrightarrow o}$ as a sub-di-operad. 
5.3. Operads from Arc families with angle markings. There are basically three approaches to generalize the actions of [K2], [K3]. The main observation is that in those actions not all boundaries were treated equally. The boundary 0 played the special role of an output. The first approach to this non-symmetric situation is to stay in the PROP setting by explicitly marking the boundaries as In or Out. This of course breaks the cyclic operad structure. The second approach, which we explain below is to put an additional angle marking on the operad which preserves the cyclic structure. The third way to proceed is to partially merge these two approaches, by keeping the In/Out distinction, and using this to define an angle marking. The angle marking is intimately linked to the operations defined by these graphs [K4].

Definition 5.8. An angle marking for an element $\alpha=(F, \Gamma, \overline{[i]}, w) \in \mathcal{A}_{g, r}^{s}$ is an angle marking $\mathrm{mk}^{<}$for $\Gamma$. This is clearly $P M C$ invariant data.

We define

$$
\mathcal{A}_{g, r}^{\angle s}:=\left\{\left(\alpha, \mathrm{mk}^{\angle}\right) \mid \alpha=(F, \Gamma, \overline{[i]}, w) \in \mathcal{A}_{g, r}^{s}, \mathrm{mk}^{\angle}: \angle_{\Gamma} \rightarrow \mathbb{Z} / 2 \mathbb{Z}\right\}
$$

to be the CW complex obtained in the analogous fashion to $\mathcal{A}_{g, r}^{s}$ where now the differential is given by deleting edges from $\left(\Gamma(\alpha), \mathrm{mk}^{\angle}\right)$ as an angle marked ribbon graph (cf. §1.2.2). We will also use notation analogous to the notation 1.3.

Notation 5.9. For all the subspaces $\&$ listed in $\S 3.1 .1$, we denote the corresponding subspaces as $\delta^{L}$. E.g. A $r c^{L}$.

Remark 5.10. $\mathcal{A} r c^{L}(n)$ is again filtered by the number of edges minus one, that is by the dimension of the cell corresponding to the graph. Now $|\angle(\alpha)|=|F(\alpha)|=$ $2|E(\alpha)|$. Let $\mathcal{A} r c^{<{ }^{k}}(n)$ be the subspace of graphs with $k+1$ edges then there is an induced exhaustive filtration

$$
\cdots \subset \mathcal{A} r c^{<\leq k}(n) \subset \mathcal{A} r c^{\angle \leq k+1}(n) \subset \cdots .
$$

We will use the identification $\mathcal{A} r c^{\angle k}(n):=\mathcal{A} r c^{<\leq k}(n) / \mathcal{A} r c^{<\leq k-1}(n)$.

Remark 5.11. $\mathcal{A} r c^{L}(n)$ has a graded open cell decomposition

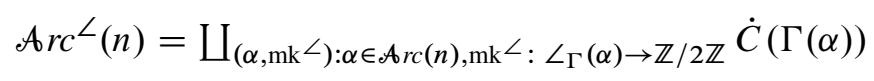

Furthermore using the angle-edge-correspondence, we have that

$$
\mathcal{A} r c_{g}^{\iota^{k}}(n)=\bigsqcup_{(\alpha): \alpha \in \mathcal{A} r c_{g}^{k}(n)} \dot{C}(\Gamma) \times(\mathbb{Z} / 2 \mathbb{Z})^{2(k+1)} .
$$

Notation 5.12. We let $\mathcal{A} r c_{\#}^{L}(n)$ be the subspaces of elements whose arc families are quasi-filling. 
Remark 5.13. $\mathcal{A} r c_{\#}^{L}(n)$ has a graded open cell decomposition

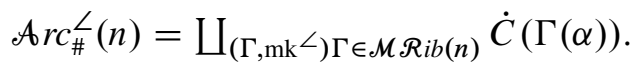

Furthermore

$$
\mathcal{A r c}_{\# g}^{L^{k}}(n)=\bigsqcup_{\Gamma \in \mathcal{M} \mathcal{R} i b_{g}^{k}(n)} \dot{C}(\Gamma) \times(\mathbb{Z} / 2 \mathbb{Z})^{2(k+1)} .
$$

5.3.1. The operadic compositions for A Arc ${ }^{\angle}$. Given $\alpha=(F, \Gamma, \overline{[i]}, w) \in \mathcal{A} r c(n)$ and $\beta=\left(F^{\prime}, \Gamma^{\prime}, \overline{\left[i^{\prime}\right]}, w^{\prime}\right) \in \mathcal{A} r c(m)$ let $\left.\alpha \circ_{i} \beta=\left(F^{\prime \prime}, \Gamma^{\prime \prime},\left[i^{\prime \prime}\right], w^{\prime \prime}\right]\right)$. We remark that using the gluing formalism of $\$ 2.2$ the angles of $\Gamma^{\prime \prime}$ were either formerly angles of $\Gamma$ and $\Gamma^{\prime}$ which we called non-split or split angles. On the one hand each split angle corresponds to a triangle in the gluing process before removing an interval in the notation of Proposition 4.2. The interval on the other hand corresponds to an angle of $\Gamma^{\prime}$ or $\Gamma^{\prime \prime}$ which is given by the two flags of the interval on the opposite triangle, we call this angle the opposite angle of the split angle. If there is a quadrangle on the opposite side, we continue the process until we hit a polygon which is not a quadrangle of $\Gamma$ of $\Gamma^{\prime}$. In both cases, when removing the intervals the identification of the corresponding flags associates a unique opposite angle to each split angle.

Given the angle markings $\mathrm{mk}^{\angle}: \Gamma \rightarrow \mathbb{Z} / 2 \mathbb{Z}$ and $\mathrm{mk}^{L^{\prime}}: \Gamma^{\prime} \rightarrow \mathbb{Z} / 2 \mathbb{Z}$ we define $\mathrm{mk}^{\angle}{ }^{\circ} \mathrm{mk}^{L^{\prime}}: \Gamma^{\prime \prime} \rightarrow \mathbb{Z} / 2 \mathbb{Z}$ as follows:

i) If $\theta \in \angle_{\Gamma^{\prime \prime}}$ is not split then the label is retained.

ii) If $\theta \in \angle_{\Gamma^{\prime \prime}}$ is split then $\theta$ will be labelled by the label of the angle opposite the split angle. (See Figure 7 for examples.)

Definition 5.14. In the above notation we define

$$
\left(\alpha, \mathrm{mk}^{\angle}\right) \circ\left(\beta, \mathrm{mk}^{L^{\prime}}\right):=\left(\alpha \circ_{i} \beta, \mathrm{mk}^{\angle} \circ_{i} \mathrm{mk}^{L^{\prime}}\right) .
$$

Remark 5.15. Using these gluings, we do not get a topological operad structure on $A r^{L}$, due to the fact that the boundaries are not behaved well with the natural differential and hence the gluings are not continuous. (see the example in Figure 7). However, the gluings are defined everywhere and are continuous up to a codimension one set.

Proposition 5.16. The above operations $\circ_{i}$ together with the $\mathbb{S}_{n}$ actions acting by permuting the labels imbue $A r^{\perp}$ with the structure of a cyclic rational operad that is almost topological. This operad structure respects the filtration $\leq k$ and hence induces an operad structure on the associated graded of the open cell decomposition. Lastly, this operad structure induces a cyclic operad structure on graded open cell level for A $r_{\#}^{\perp}$ and hence on the set of Abelian groups $\mathcal{R}_{i b}{ }^{\perp}$ which are isomorphic to the graded open cell decomposition of $\mathcal{A r c}_{\#}^{L}$. 

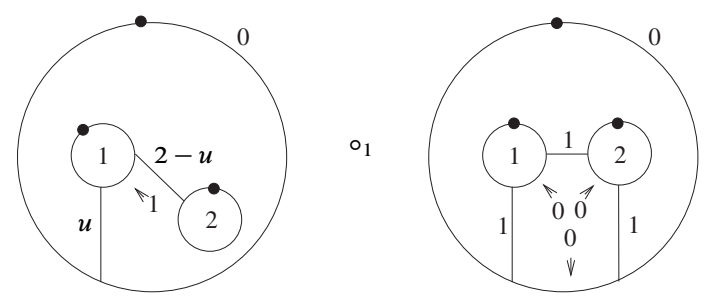

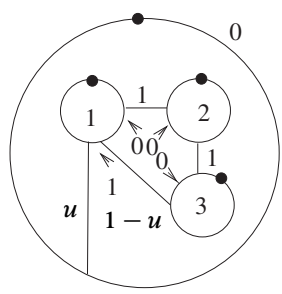

$u<1$

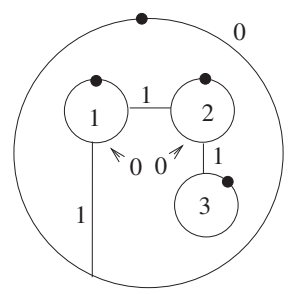

$u=1$

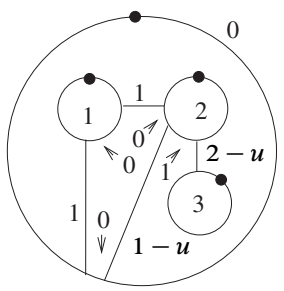

$u>1$

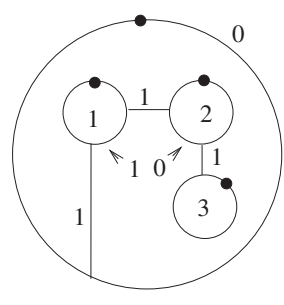

$\lim u>1, u<1$

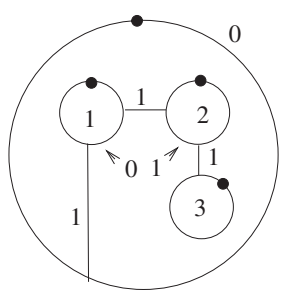

$\lim u>1, u>1$

Figure 7. An example showing that $\mathcal{A} r c^{\angle}$ is only a rational topological operad.

Proof. The fact that the $\circ_{i}$ yield an operad of sets follows from the associativity of the operad structure on $\mathcal{A r c}$ and the associativity of the marking function under the composition. It is clear that the operad structure is continuous on the interior of the cells and possibly discontinuous only on the boundaries which are at least codimension one. Thus we obtain a rational operad structure that is almost topological. Since the operad structure on $\mathcal{A} r c$ respects the filtration, so does the operad structure on $\mathcal{A r c}{ }^{L}$. The other facts are now straightforward.

5.3.2. De-projectivized version and extended gluings. As was the case for $\mathcal{A}$ and Arc there are straightforward generalizations of the gluing operations to $A^{L} \times \mathbb{R}_{>0}$ and to the analogue of $\mathscr{D} \mathcal{A}$ and moreover extensions on these sets involving gluing to empty boundaries. The constructions are mutatis mutandis the same as in $\$ 2.4$ and $\$ 3.2 .1$. 
5.3.3. Standard markings and suboperads. Notice that for an element $\alpha \in \mathcal{A} r c$, $\alpha=(F, \Gamma, \overline{[i]}, w)$ there are two types of angles. The angles $(f, \curvearrowright(f))$ in which $f<(\curvearrowright(f))$ in the natural linear order $<$ at the boundary of $f-$ we will call these angles the inner angles - and the angles $(f, \curvearrowright(f))$ in which $f>(\curvearrowright(f))$ which we will call outer angles. There is exactly one outer angle at each boundary.

There are several embeddings $\mathcal{A} r c \subset \mathcal{A} r c^{L}$. Each one is given by choosing a function $\mathrm{mk}^{L}$. Three of these choices are rather canonical. The first is by a constant marking of all angles by 0 , the second is given by the constant angle marking 1 . Lastly, one can mark all inner angles by 0 and the outer angles by 1 .

All these markings are interesting and embed $\mathcal{A} r c$ as a cyclic operad; that is the image is truly a cyclic operad and not just a rational operad. The second marking will play a special role for us in [K4] as it leads to a connection with the $\Xi_{2}$ operad of [MS3]. So for $\alpha \in \mathcal{A}$ we define the standard marking to be given by

$$
\mathrm{mk}^{\angle}(\theta) \equiv 1
$$

Caveat. For the non-cyclic suboperads $\mathcal{T}$ ree and $\mathscr{L} \mathcal{T}$ ree there are two standard markings one as suboperads of $\mathcal{A} r c$, and the second as sub-di-operads of $\mathcal{A} r \mathrm{CC}^{i / o}$ as defined in the next paragraph.

The standard marking $\mathrm{mk}^{L}$ for $\mathcal{A} \mathrm{c}^{i / o}$ is given by

$$
\mathrm{mk}^{L}(\theta)= \begin{cases}1 & \text { if } \theta \text { is an outer angle, } \\ 1 & \text { if } \theta \text { is an inner angle belonging to an In boundary, } \\ 0 & \text { if } \theta \text { is an inner angle belonging to an Out boundary. }\end{cases}
$$

This also gives standard markings for $\mathcal{T}$ ree and $\mathscr{L} \mathcal{T}$ ree, when considering the boundary 0 as the only Out boundary and the other boundaries as In boundaries.

Notation 5.17. Sometimes it will be necessary to distinguish between an arc graph and the arc graph with standard angle markings. To facilitate this distinction, for an arc graph $\alpha$ we write $\alpha^{L}$ for the arc graph with the standard marking. Here the standard marking for $\mathcal{A} r c$ is defined by (5.6) and the standard marking for $\mathcal{A} r c^{i / o}$ is defined by (5.7).

Lemma 5.18. The spaces Arc, its suboperads $\mathcal{T}$ ree, $\mathscr{L} \mathcal{T}$ ree and their restrictions $c p$ with the above marking (5.6) or any of the other markings discussed in $\$ 5.3 .3$ are suboperads of $\mathcal{A} r c^{L}$.

The subspace and $\mathcal{A} r c^{i \leftrightarrow o}$ (and hence $\mathcal{T}$ ree and $\mathscr{L} \mathcal{T}$ ree viewed as in $\$ 5.2 .1$ with the convention (5.7)), are partial suboperads. Here the partial compositions are dictated by the marking $\mathrm{mk}^{<}$. A boundary is an In boundary if one and hence all of its inner 
angles are marked by 1 and a boundary is an out boundary if one and hence all of its inner angles are marked by 0 . The partial operad structure is given by restricting the operadic composition to connecting In to Out boundaries only.

Proof. The claim for $\mathcal{A} r c$ and hence its suboperads, is clear, since all markings will always be constantly 1 in the standard marked case. In the other cases either we have a constant marking or only the outer angles are marked by 1 . These latter marking is invariant by the definition of deleting edges in a tree with angle markings as defined in $\S 1.2 .2$.

For $\mathcal{A} r c^{i \leftrightarrow o}$ first notice that the outer angles are always non-split, so they retain their marking by 1 . Thus in the case of $\mathcal{A} r \mathrm{Cc}^{i \leftrightarrow o}$, we only have to look at split angles. In the case an angle is split then the opposite angle is on a boundary with the same In/Out marking due to the definition of $\mathcal{A} r c^{i \leftrightarrow o}$.

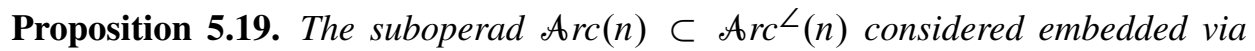
(5.6) or any of the other embeddings of 5.2.1 is a topological suboperad. And $\mathcal{A} r c^{i \leftrightarrow o}(n, m) \subset A r c^{\perp}(n+m-1)$ is a cyclic partial topological operad that defines a di-operad in any of the above embeddings.

Proof. The only problems that can arise are on the boundary. The compatibility of the different limit for these subspaces follows from the definition of deleting edges in angle marked graphs. In particular, for $\mathcal{A} r c$ this observation is trivial, since the angle marking is constant and stays constant. For $\mathcal{A} r c^{i \leftrightarrow o}$ notice that since the taking a limit into the boundary by deleting an arc corresponds to deleting an edge in the dual graph. The rule for the boundary marking is given by $\bar{a} \bar{b}$. If there is an outer angle involved, then we have no problem since $\overline{\overline{1}} \bar{a}=\overline{0}=1$ and the merged angle is again an outer angle. On inner angles, we always have $a \equiv b$, so that $\overline{\bar{a}} \bar{b}=a$. In other words, the limit obtained by deleting edges from the angle marked graphs reproduces the standard marking. The partial operad structure is cyclic and allows to glue one "in" to one "out", so by definition it gives rise to a di-operad structure.

Corollary 5.20. The PL-chain level operads of $\mathcal{A} r c, \mathcal{A} r c^{i \leftrightarrow o}$ thought of as topological suboperads of the chains of $\mathrm{Arc}^{\perp}$ are dg-chain operads.

Just as in the case of $\mathcal{A} r c$ there are analogues of the cell complexes and quasi-filling sub-complexes:

Proposition 5.21. The relative chains $\mathcal{C}_{o}^{*}\left(\mathcal{A} r \mathrm{c}^{<}\right)$form an operad. The quasi-filling analogues $A r_{\# \#}$ and $\mathcal{A} r c_{\#}^{i \leftrightarrow o}$ and also the associated graded relative chain complexes of these spaces form dg operads.

Proof. These statements follows directly from the operad structure of $\mathcal{A r c}^{<}$. Namely, we can write the degree $k$ component of $\mathcal{A} r c^{L}(n)$ as $\mathcal{A} r c^{L^{k}}(n)=\mathcal{A} r c(n)^{k} \times$ 
$(\mathbb{Z} / 2 \mathbb{Z})^{2(k+1)}$. We also know that the composition respects the filtration of $\mathcal{A r c}^{\perp}$ and the factor $(\mathbb{Z} / 2 \mathbb{Z})^{2(k+1)}$ can be thought of as indexing the cells by discrete data. So the statements follow from the fact that the analogous statements are true for Arc. $\square$

5.4. Local scaling and "up to homotopy" structures. The di-operad $\mathcal{A r c}^{i / o}$ falls short of being a PROP, since we can only guarantee the compatibility of the weights for one boundary by using suitable representatives in the projective class. If we are willing to relax the associativity up to homotopy, we can, however, achieve a quasiPROP structure on $\mathcal{A} r c^{i \leftrightarrow i}$. For this we will introduce a new composition which is given by a local scaling, since globally, we can in general only scale to match one boundary. Now we will scale only the arcs incident to the two boundaries which are to be glued. This will destroy the associativity, but the associativity does hold up to homotopy so that there will be an honest associative structure on the homology level and in some situations, with a careful choice of chains, also on the chain level.

Definition 5.22. A quasi-operad is an operad in which the axiom of associativity need not hold and a quasi-PROP is a PROP is which the axiom of associativity need not hold.

A quasi-operad/PROP is called a homotopy operad/PROP, if it is in the category of topological spaces and the associativity equations (respectively the compatibility equations) hold up to homotopy.

5.4.1. The Sullivan quasi-PROP. Notice that for an element $\alpha \in \mathcal{A} r c^{i \leftrightarrow i}$, we can independently scale the weights of the arcs running to the inputs, since there are no arcs which go from one input to another input. Therefore the set of all arcs are in a 1-1 correspondence to the union of the sets of arcs incident to the In boundaries. In order to glue the inputs to outputs, using this bijection we first scale the weights on the input factors locally - that is separately for each boundary homogeneously we scale all the weights of edges incident to that boundary - to make them match with the output weights and then glue. More precisely, let $\alpha \in \mathcal{A} r c^{i \leftrightarrow i}(n, m)$ and $\beta \in \mathcal{A} r c^{i \leftrightarrow i}(m, k)$. Let $\operatorname{Out}(\alpha)$ be the indexing set of the "out" boundaries of $\alpha$ and $\operatorname{In}(\beta)$ be the indexing set of the "in" boundaries of $\beta$. For $j \in \operatorname{Out}(\alpha)$ and $i \in \operatorname{In}(\beta)$ let $w_{j}$ be the sum of the weights incident to the boundary $j$ of $\alpha$ and similarly let $w_{i}$ be the sum of the weights of the arcs incident to the boundary $i$ of $\beta$. Let $\phi: \operatorname{Out}(\alpha) \rightarrow \operatorname{In}(\beta)$ be the bijection for the gluing. Then we define $\alpha \bullet_{\phi} \beta$ to be the arc family obtained by first scaling the arcs incident to the boundary $i$ of $\beta$ by the factor $\frac{w_{j}}{w_{i}}$ (where $j=\phi^{-1}(i)$ ), and then gluing the arcs of $\alpha$ and $\beta$ along the boundaries $i$ and $j$ which now have the same total incident weight.

To define the vertical compositions one can use disjoint union of surfaces by passing to possibly disconnected surfaces.

Theorem 5.23. The operations $\bullet_{\phi}$ imbue the sets generated by $\mathbb{S}_{n} \times \mathbb{S}_{m}$ modules $\mathcal{A r c}{ }^{i \leftrightarrow i}(n, m)$ with the structure of a homotopy PROP. 
Proof. Tedious, but straightforward. The main observation is that if one lets $C_{i}$ be the images of the boundaries after the gluing of the surfaces, each of the glued arcs can be moved to transversally cut exactly one of these curves. Now there are commuting flows that act locally at $C_{i}$ which essentially move the weights of the arcs before gluing. The example of one boundary gluing is given in [KP]. The flow is a generalization of that of [K1] and given by moving from one partial homotopy diagonal to another. These flows allow one to flow from one association to the other.

Corollary 5.24. The homology of $\mathcal{A} r c^{i \leftrightarrow i}(n, m)$ is a PROP.

Notation 5.25. We phrased the theorem so that we can avoid yet other notation. What is meant is that the operations $\bullet_{\phi}$ and the $\mathbb{\$}_{n} \times \mathbb{S}_{m}$ actions naturally induce operations and permutation actions on the PROP whose $(N, M)$-component is given by

$$
\bigsqcup_{\left(n_{1}, \ldots, n_{k}\right), \sum n_{i}=N ;\left(m_{1}, \ldots m_{k}\right), \sum n_{j}=M} \prod_{n_{i}, m_{j}} \mathcal{A} r c^{i \leftrightarrow i}\left(n_{i}, m_{i}\right) .
$$

We will call this PROP simply $\mathcal{A} r c^{i \leftrightarrow i}$ and also use this shorthand notation for any sub-PROP generated by a subset of $\mathcal{A} r r^{i \leftrightarrow i}$.

Remark 5.26. This scaling when restricted to $\mathcal{T}$ ree or $\mathscr{L} \mathcal{T}$ ree is the quasi-operad structure used for the normalized versions of cacti and spineless cacti [K1].

Remark 5.27. If one wishes to avoid disconnected surfaces, one can use the following trick. Let $I(m, m) \in \mathcal{A} r c^{i \leftrightarrow o}(m, m)$ be the surface of genus 0 with $2 m$ boundaries which are partitioned into two sets In and Out which are individually numbered from 1 to $m$ with exactly one arc running from the in boundary $i$ to the out boundary $i$. In this case, we define the vertical PROP composition $\alpha \otimes \beta$ for $\alpha \in \mathcal{A} r c^{i \leftrightarrow i}(m, n)$ and $\beta \in \mathcal{A} r c^{i \leftrightarrow i}(k, l)$ to be given by the gluing of the out boundaries of $\alpha$ onto the first $n$ In boundaries of $I(n+l, n+l)$ and gluing the Out boundaries of $\beta$ to the last $l$ of the In boundaries of $I(n+l, n+l)$.

5.5. Cell models. Without reiterating all the details of the construction, it is clear that there are induced (quasi)-operads, PROPs and di-operads on the graded open cell level. These structures can be seen to be strict on the this level. If we pass to the graded cells also the rational operad structure becomes a strict operad structure. We keep the convention in the PROP gluing that is analogous to equation 2.7 , namely that the operation on cells is zero if for any of the pairs of boundaries which are glued in the PROP operation both boundaries are twisted.

Theorem 5.28. On the (graded) open cell level the homotopy (or rational) structures give rise to the non-homotopy structures, moreover the quasi-filling subspaces are isomorphic to types of graphs and hence there is the induced structure on these graphs. In particular, we have: 


\begin{tabular}{l|l} 
& is a \\
\hline $\mathcal{C}_{o}^{*}\left(\mathcal{A} r c^{i / o}\right)$ & di-operad \\
$\mathcal{C}_{o}^{*}\left(\mathcal{A} r c^{i \leftrightarrow i}\right)$ & sub-di-operad and a PROP \\
$\bigodot_{o}^{*}\left(\mathcal{A} r c^{i \leftrightarrow o}\right)$ & sub-di-operad and a sub-PROP
\end{tabular}

Furthermore going to the graded cells, we obtain operads which are isomorphic to the free Abelian groups of certain types of ribbon graphs:

\begin{tabular}{|c|c|c|}
\hline & is a & and is isomorphic to \\
\hline $\mathrm{Gr} \mathcal{C}_{o}^{*}\left(\mathcal{A} r c^{\perp}\right)$ & cyclic operad & \\
\hline $\operatorname{Gr} \bigodot_{o}^{*}\left(A r c_{\#}^{L}\right)$ & cyclic operad & $\mathcal{R} i b^{L}$ \\
\hline $\operatorname{Gr} \mathscr{C}_{o}^{*}\left(\mathcal{A} r c_{\#}\right)$ & dg cyclic operad & $\mathcal{R i b}$ \\
\hline $\operatorname{Gr} \mathcal{C}_{o}^{*}\left(\mathcal{A} r c_{\#}^{i \leftrightarrow o}\right)$ & $d g-P R O P$ & $\mathcal{R} i b^{i \leftrightarrow o}$ \\
\hline
\end{tabular}

Here the table is to be understood in the sense that the entry in the first column carries the structure of the second column and is additively (over $\mathbb{Z}$ ) isomorphic to the third column thereby inducing the respective structure.

Proof. The claims for the di-operad structures follow for the results about Arc. For the PROP structure, the arguments are analogous to those of \$2.3.2. We again claim that the PROP operations are 1-1 on cells which are not twisted at the boundary. The case of closed loops cannot appear. After scaling, the gluing operation is locally given by the shuffle combinatorics of $\$ 2.2$, so that indeed the image of the PROP action will be full cells and 1-1 in the case that not both the boundaries are twisted. This local argument accounts for all arcs of the glued surface passing through the images of the curves defined by the glued boundaries whose weights can all be scaled independently, while all other arcs are unchanged. So indeed we get an induced map on cells. Locally, that is regarding each pair of glued boundaries separately, the map is 1-1 precisely if the two boundaries are not both twisted and there are no closed loops. This yields the above assertion. The fact that the image cells lie in the relevant PROP is clear by the definition of the restriction. Combining two "in" to "out" arcs yields an arc of the same type, and also combining an "out" to "out" arc with an "in" to "out" arc again yields an arc running from "out" to "out". For the rational structures, we notice that again all "problems" arise in at most codimension one. Consequently passing to the associated graded induces the unrestricted structures.

5.6. A CW model for Sullivan chord diagrams. Recall that $\mathcal{A}$ was the $\mathrm{CW}$ complex that contains $\mathcal{A} r c$ as a subset. We define $\mathcal{A}_{g, r}^{s i / o}:=\mathcal{A}_{g, r}^{s} \times(\mathbb{Z} / 2 \mathbb{Z})^{r}$ analogously to $\mathcal{A r c}{ }^{i / o}$. Notice that these spaces are $\mathrm{CW}$-complexes. We call the collection of these spaces $\mathcal{A}^{i / o}$ and as usual write $\mathcal{A}^{i / o}(n):=\bigsqcup_{g, s} A_{g, n+1}^{s i / o}$. 
Definition 5.29. We let $\overline{\mathcal{A r c}}^{i \leftrightarrow i}$ be the collection of subspaces of the spaces of $\mathcal{A}^{i / o}$ in which there are only arcs running from the In to the Out and possibly from the Out to the Out boundaries and there is no empty In boundary.

We let $\overline{\mathcal{A r C}}^{i \leftrightarrow o} \subset \overline{\mathrm{A} r c}^{i \leftrightarrow i}$ be the subset where arcs only run from the In boundaries to the In boundaries.

We also let $\overline{\mathscr{L} \mathcal{A} r c}{ }^{i \leftrightarrow i}$ be the subset of elements whose underlying arc graph is not

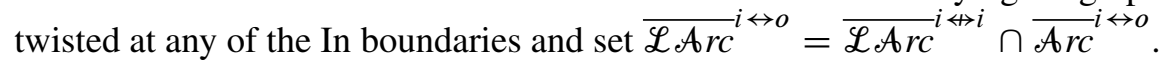

Remark 5.30. It is clear that $\overline{\mathcal{A r c}}^{i \leftrightarrow o}$ is a retract of $\overline{\mathcal{A r c}}^{i \leftrightarrow i}$ by simply scaling all the weights on the arcs connecting Out to Out to zero. So any cell model for $\overline{\mathcal{A} r c} i \leftrightarrow o$ also yields a cell model for $\overline{\mathcal{A} r c} i \leftrightarrow i$.

5.6.1. Extended gluing on $\overline{\mathcal{A r c}}^{i \leftrightarrow o}$. We can extend the operadic gluing to $\mathcal{A}^{i / o}$ as in $\$ 3.2 .1$, in particular for $\overline{\mathcal{A r c}}^{i \leftrightarrow i}$ this means that if we glue to an empty "out" boundary, the arcs incident to the respective "in" boundary will be deleted. Notice that this leaves us in $\overline{\mathcal{A r c}}^{i \leftrightarrow i}$ since none of the arcs hitting the "in" boundaries in the glued surface have been effected. As mentioned earlier this operation is only associative up to homotopy, so $\overline{A r c}^{i \leftrightarrow i}$ is a quasi-di-operad. With respect to the PROP structure we define the maps $\bullet_{\phi}$ by setting the weights of the arcs incident to an "in" boundary that is glued to an empty "out" boundary to zero. These gluings then goes over to the cell level as in $\$ 2.3$ and Theorem 5.28.

In particular, this yields the following extension of the gluing to $\bigodot_{o}^{*}\left(\overline{\mathcal{A r c}}^{i \leftrightarrow i}\right)$. The gluing of a cell indexed by an arc graph with an empty "out" glued at that "out" to a non-empty "in" is defined to be the cell indexed by the modified image of the two arc graphs, where the modification is that in the second arc graph all edges incident to the boundary have been deleted as detailed in 2.4.

Just like for cacti and spineless cacti [K1], there is a smaller space which is a retract of $\overline{\mathcal{A r c}}^{i \leftrightarrow o}$ that is actually a CW complex.

Definition 5.31. We define $\bar{A} \boldsymbol{A r c}_{1}^{i \leftrightarrow o} \subset \overline{\mathcal{A r c}}_{1}^{i \leftrightarrow o}$ to be the subspace of graphs whose sum of weights of arcs incident to every In boundary vertex is one.

It is clear that $\overline{\mathcal{A r c}}^{i \leftrightarrow o}$ retracts to $\overline{\mathcal{A r c}}_{1}^{i \leftrightarrow o}$ by homogenously scaling the weights of the sets of arcs incident to each In boundary separately for each of these sets, so that their total weight becomes one.

Proposition 5.32. $\overline{A r c}_{1}^{i \leftrightarrow o}$ is a CW complex, whose cells are indexed by the arc graphs of the given type. 
Proof. Completely analogous to the constructions of [K1], [K2]. Given an arc graph, we define the cell by $\times_{v \in \operatorname{In}} \Delta^{|v|}$ where $\Delta$ is the standard simplex. We define the attaching maps by gluing the boundary corresponding to a face of a simplex to the cell of lower dimension indexed by the arc graph obtained form the original arc graph by deleting exactly the edge that indexes the face. It is then straightforward to show that this $\mathrm{CW}$ complex realizes $\overline{\mathrm{Arc}}_{1}^{i \leftrightarrow o}$.

It is clear that the graphs of $\overline{\mathscr{L A A r c}}^{i \leftrightarrow o}$ form a sub-CW complex of $\overline{\mathcal{A C c}}_{1}^{i \leftrightarrow o}$ which we call $\overline{\mathscr{L} A r c}_{1}^{i \leftrightarrow o}$.

We define the di-operadic compositions on $\overline{A r r c}_{1}^{i \text { «i }}$ by scaling the input individually to the weight of the output. The homotopy PROP structure is just the homotopy sub-PROP structure. Notice that in the gluings $\bullet_{\phi}$ one only scales at the In boundaries which are to be glued so that the weights on the In boundaries which remain after gluing are unchanged.

Theorem 5.33. These compositions define a homotopy-PROP structure on $\overline{\mathcal{A r c}}_{1}^{i \leftrightarrow i}$

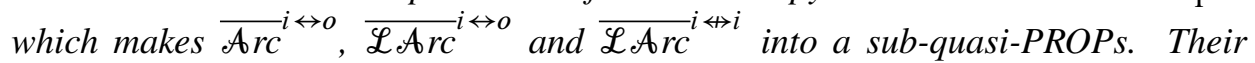
homotopy-PROP structures descend to PROP structures on $C_{o}^{*}\left(\overline{\mathcal{A r C}}^{i \ll i}\right)$ and $\operatorname{Gr} \mathcal{C}_{o}^{*}\left(\overline{\mathcal{A r C}}^{i \leftrightarrow i}\right)$. Also, the PROP structure of $\overline{\mathcal{A r c}}_{1}^{i \leftrightarrow o}$ descends to a dg-PROP structure on $C C_{*}\left(\overline{\mathcal{A r C}}_{1}^{i \leftrightarrow o}\right)$. Moreover, the two PROP structures $C C_{*}\left(\overline{\mathcal{A r C}}_{1}^{i \leftrightarrow o}\right)$ and $\mathrm{Gr}_{o}^{*}\left(\overline{\mathcal{A r C}}^{i \leftrightarrow o}\right)$ and their differentials when viewed as defined on the same free Abelian group generated by the respective arc graphs agree.

The same statement mutatis mutandis holds true for the respective di-operad structures. Likewise the analogous statements hold true for $\overline{\mathscr{L A t r}}^{i \leftrightarrow o}$ and $\overline{\mathscr{L A C r}}_{1}^{i \leftrightarrow o}$ as well.

Proof. Tedious but straightforward generalization to the case of several outputs of the analogous statement about $\mathcal{C}_{a c t i}{ }^{1}$ and $\boldsymbol{C}_{\text {acti }}$ given in [K1]. Since we are dealing with a local scaling which acts independently on the arcs due to the restrictions we imposed, one can construct a homotopy which scales the sum of the weights of the arcs through any given fixed closed curve which is the image of a boundary under a gluing to say 1. Using these homotopies, one can flow from one association to the other, this shows the quasi-PROP structure. On the cell level, one has to make sure that all the combinatorially possible graphs arise and that each weight only arises once. This is a straightforward verification using the techniques presented above. The subPROPs are actually stable under gluing, since one will never induce a twist on the In boundaries when gluing. Likewise one cannot obtain any "out" to "out" arcs if they were not previously there. The last statement about the CW-complex follows from the fact that the cells of lower dimension are killed in the cellular chain complex. 
Corollary 5.34. The $\mathbb{S}_{n} \times \mathbb{S}_{m}$-modules $C C_{*}\left(\overline{\mathcal{A r c}}_{1}^{i \leftrightarrow o}\right)(n, m)$ form a $d g$-PROP and give a chain model operad for $\overline{A r c} i \leftrightarrow o$ that is for the extended metric Sullivan chord diagrams.

The same statement holds true for the respective di-operad structures.

Corollary 5.35. $H_{*}\left(\overline{\mathcal{A r C}}_{1}^{i \leftrightarrow o}\right) \simeq H_{*}\left(\overline{\mathcal{A r C}}^{i \leftrightarrow o}\right)$ and the induced PROP structures

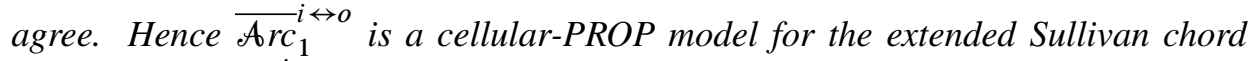
PROP Gr $\mathcal{C}_{o}^{*}\left(\overline{\mathcal{A} r c}^{i \leftrightarrow o}\right)$.

The same statement holds true for the respective di-operad structures.

\section{References}

[BF] C. Berger and B. Fresse, Une décomposition prismatique de l'opérade de BarrattEccles. C. R. Acad. Sci. Paris Sér. I Math. 335 (2002), 365-370. Zbl 1016.18005 MR 1931518

[CS] M. Chas and D. Sullivan, String topology. Ann. of Math., to appear; preprint 1999, arXiv:math.GT/9911159

[Ch] D. Chataur, A bordism approach to string topology. Internat. Math. Res. Notices 2005 (2005), 2829-2875. Zbl 1086.55004 MR 2180465

[C] R. L. Cohen, Multiplicative properties of Atiyah duality. Homology Homotopy Appl. 6 (2004), 269-281. Zbl 1072.55004 MR 2076004

[CG] R. L. Cohen and V. Godin, A polarized view of string topology. In Topology, geometry and quantum field theory (Oxford, 2002), London Math. Soc. Lecture Note Ser. 308, Cambridge University Press, Cambridge, 2004, 127-154. Zbl 1095.55006 MR 2079373

[CJ] R. L. Cohen and J. D. S. Jones, A homotopy theoretic realization of string topology. Math. Ann. 324 (2002), 773-798. Zbl 1025.55005 MR 1942249

[CV] J. Conant and K. Vogtmann, On a theorem of Kontsevich. Algebr. Geom. Topol. 3 (2003), 1167-1224. Zbl 1063.18007 MR 2026331

[Co] K. J. Costello, Topological conformal field theories and Calabi-Yau categories. Preprint 2004, arXiv:math.QA/0412149; The Gromov-Witten potential associated to a TCFT, preprint 2005, arXiv:math.QA/0509264; A dual point of view on the ribbon graph decomposition of moduli space, preprint 2006, arXiv:math.GT/0601130

[H] J. L. Harer, Stability of the homology of the mapping class groups of orientable surfaces. Ann. of Math. (2) 121 (1985), 215-249. Zbl 0579.57005 MR 786348

[HM] J. Hubbard and H. Masur, Quadratic differentials and foliations. Acta Math. 142 (1979), 221-274. Zbl 0415.30038 MR 523212

[J] J. D. S. Jones, Cyclic homology and equivariant homology. Invent. Math. 87 (1987), 403-423. Zbl 0644.55005 MR 870737 
[KLi1] A. Kapustin and Y. Li, D-branes in Landau-Ginzburg models and algebraic geometry. J. High Energy Phys. 2003, no. 12, 005. MR 2041170

[KLi2] A. Kapustin and Y. Li, Topological correlators in Landau-Ginzburg models with boundaries. Adv. Theor. Math. Phys. 7 (2003), 727-749. Zbl 1058.81061 MR 2039036

[KR] A. Kapustin and L. Rozansky, On the relation between open and closed topological strings. Comm. Math. Phys. 252 (2004), 393-414. Zbl 1102.81064 MR 2104884

[K1] R. M. Kaufmann, On several varieties of cacti and their relations. Algebr. Geom. Topol. 5 (2005), 237-300. Zbl 1083.55007 MR 2135554

[K2] R. M. Kaufmann, On Spineless Cacti, Deligne's Conjecture and Connes-Kreimer's Hopf Algebra. Preprint 2003, arXiv:math.QA/0308005

[K3] R. M. Kaufmann, A proof of a cyclic version of Deligne's conjecture via Cacti. Preprint 2004, arXiv:math.QA/0403340

[K4] R. M. Kaufmann, Moduli space actions on the Hochschild co-chains of a Frobenius algebra II: correlators. Preprint 2006, arXiv:math/0606065

[K5] R. M. Kaufmann, The arc spectrum. In preparation.

[KLP] R. M. Kaufmann, M. Livernet, and R. C. Penner, Arc operads and arc algebras. Geom. Topol. 7 (2003), 511-568. Zbl 1034.18009MR 2026541

[KP] R. M. Kaufmann and R. C. Penner, Closed/open string diagrammatics. Nuclear Phys. $B 748$ (2006), 335-379. MR 2242677

[Ko1] M. Kontsevich, Intersection theory on the moduli space of curves and the matrix Airy function. Comm. Math. Phys. 147 (1992), 1-23. Zbl 0756.35081 MR 1171758

[Ko2] M. Kontsevich, Formal (non)commutative symplectic geometry. In The Gel'fand Mathematical Seminars, 1990-1992, Birkhäuser, Boston 1993, 173-187. Zbl 0821.58018 MR 1247289

[Ko3] M. Kontsevich, Operads and motives in deformation quantization. Lett. Math. Phys. 48 (1999), 35-72. Zbl 0945.18008 MR 1718044

[KS1] M. Kontsevich and Y. Soibelman, Deformations of algebras over operads and the Deligne conjecture. In Conférence Moshé Flato 1999 (Dijon), Vol. I, Math. Phys. Stud. 21, Kluwer Academic Publishers, Dordrecht 2000, 255-307. Zbl 0972.18005 MR 1805894

[KS2] M. Kontsevich and Y. Soibelman, Notes on $A_{\infty}$-categories and non-commutative geometry. Manuscript.

[MS1] J. E. McClure and J. H. Smith, A solution of Deligne's Hochschild cohomology conjecture. In Recent progress in homotopy theory (Baltimore, MD, 2000), Contemp. Math. 293, Amer. Math. Soc., Providence, RI, 2002, 153-193. Zbl 1009.18009 MR 1890736

[MS2] J. E. McClure and J. H. Smith, Multivariable cochain operations and little $n$-cubes. J. Amer. Math. Soc. 16 (2003), no. 3, 681-704 Zbl 1014.18005 MR 1969208

[MS3] J. E. McClure and J. H. Smith, Cosimplicial objects and little $n$-cubes. I. Amer. J. Math. 126 (2004), 1109-1153. Zbl 1064.55008 MR 2089084

[Me] S. A. Merkulov, De Rham model for string topology. Internat. Math. Res. Notices 2004 (2004), 2955-2981. Zbl 1066.55008 MR 2099178 
[P] R. C. Penner, The decorated Teichmüller space of punctured surfaces. Commun. Math. Phys. 113 (1987), 299-339. Zbl 0642.32012 MR 0919235

[P2] R. C. Penner, Decorated Teichmüller theory of bordered surfaces. Comm. Anal. Geom. 12 (2004), 793-820. Zbl 1072.32008 MR 2104076

[St] K. Strebel, Quadratic differentials. Ergeb. Math. Grenzgeb. (3) 5, Springer-Verlag, Berlin 1984. Zbl 0547.30001 MR 0743423

[S1] D. Sullivan, Open and closed string field theory interpreted in classical algebraic topology. In Topology, geometry and quantum field theory (Oxford, 2002), London Math. Soc. Lecture Note Ser. 308, Cambridge University Press, Cambridge 2004, 344-357. Zbl 1088.81082 MR 2079379

[S2] D. Sullivan, Sigma models and string topology. In Graphs and patterns in mathematics and theoretical physics, Proc. Sympos. Pure Math. 73, Amer. Math. Soc., Providence, RI, 2005, 1-11. Zbl 1080.53085 MR 2131009

[T] D. E. Tamarkin, Another proof of M. Kontsevich formality theorem. Peprint 1998, arXiv:math/9803025; Formality of chain operad of little discs. Lett. Math. Phys. 66 (2003), 65-72. Zbl 1048.18007 MR 2064592

[TZ] T. Tradler and M. Zeinalian, On the cyclic Deligne conjecture. J. Pure Appl. Algebra 204 (2006), 280-299. Zbl 02242211 MR 2184812

[V] A. A. Voronov, Homotopy Gerstenhaber algebras. In Conférence Moshé Flato 1999 (Dijon), Vol. II, Math. Phys. Stud. 22, Kluwer Academic Publishers, Dordrecht 2000, 307-331. Zbl 0974.16005 MR 1805923

[V2] A. A. Voronov, Notes on universal algebra. In Graphs and patterns in mathematics and theoretical physics, Proc. Sympos. Pure Math. 73, Amer. Math. Soc., Providence, RI, 2005, 81-103. Zbl 1083.18005 MR 2131012

Received September 4, 2006; revised March 15, 2007

R. M. Kaufmann, Department of Mathematics, University of Connecticut, Storrs, CT 06269-3009, U.S.A.

E-mail: kaufmann@math.uconn.edu 